\title{
Análises estatísticas de alturas significativas de ondas de série temporal de curto prazo na costa do Rio Grande do Norte
}

\author{
Maria de Fátima Alves de MATOS ${ }^{1}$, Venerando Eustáquio AMARO², Ada Cristina \\ SCUDELARI ${ }^{3}$ \& Ana Cleide Nascimento BEZERRA ${ }^{4}$ \\ 1 Programa de Pós-graduação em Engenharia Civil, Universidade Federal do Rio Grande do Norte. Av. \\ Salgado Filho, s/n, CEP 59.540-000, Natal, RN, Brasil (fatima.alves@ct.ufrn.br). \\ 2 Departamento de Engenharia Civil, Programa de Pós-graduação em Engenharia Civil, Universidade \\ Federal do Rio Grande do Norte (venerando.amaro@gmail.com). \\ 3 Departamento de Engenharia Civil, Programa de Pós-graduação em Engenharia Civil, Universidade \\ Federal do Rio Grande do Norte (ada@ct.ufrn.br). \\ ${ }^{4}$ Programa de Pós-graduação em Engenharia Civil, Universidade Federal do Rio Grande do Norte \\ (anabezerra@ct.ufrn.br).
}

\begin{abstract}
Resumo. O objetivo deste trabalho é apresentar uma nova abordagem do clima de ondas disponível para a costa norte do Rio Grande do Norte, introduzindo análises espacial e temporal relacionadas a uma série temporal dos últimos cinco anos. 0 estudo fornece primeiramente uma avaliação da distribuição espacial das alturas significativas das ondas ao longo de uma série temporal entre 2010 a 2015, com análise anual, sazonal e mensal dos padrões de altura significativa (Hs) e direções médias (DIR) em todo o domínio espacial. Na segunda etapa, as variações temporais são analisadas considerando as médias mensais, a variabilidade sazonal e as variações interanuais nas estações costeiras. Finalizando, o estudo oferece uma classificação dos eventos severos de ondas a partir da aplicação da técnica dos percentis, cujo valor $=P_{0,99}$ foi definido para estabelecer o limiar fixo em relação ao valores de Hs da série temporal. $A$ distribuição anual e sazonal das alturas significativas das ondas no domínio espacial mostra que a zona de maior incidência, com os valores mais elevados está localizada na porção externa da plataforma continental, acima da isóbata de 25 m, com média de 1,96 m e valores máximos acima de $2 \mathrm{~m}$ para Hs com padrão direcional de ENE. Nas análises sazonais, durante os meses de verão as ondas são as mais altas nesta costa. Nas análises sazonais e interanuais, os anos de 2010 e 2013 foram considerados os anos de maior agitação e com maior frequência dos eventos severos na região. 0 estudo vem corroborar tanto no conhecimento dos padrões das ondas quanto na possibilidade de avaliar as ondas como recurso energético neste setor da costa do Nordeste.
\end{abstract}

Palavras-chave. Ondas de superfície, série temporal, modelagem, sazonalidade, percentil, RN.

\begin{abstract}
STATISTICAL ANALYSIS OF SIGNIFICANT HEIGHTS OF SHORT-TERM SEASONAL SERIES WAVES AT THE COAST OF THE RIO GRANDE DO NORTE. This work aims to present a new approach of wave climate available for the North coast of Rio Grande do Norte, introducing spatial and temporal analyses related to a time series of the last five years. The study provides a first evaluation of the spatial distribution of significant wave heights over a temporal series between 2010 to 2015, with annual, seasonal and monthly analysis of the patterns of significant height (Hs) and average directions (DIR) throughout the spatial domain. The temporal variations are analyzed in the second step, considering the monthly averages, the seasonal variability and inter-annual variations at the coastal stations. Finally, the study offers a classification of severe wave events based on the application of the percentile technique, which percentile $=P_{0.99}$ was set to establish a fixed threshold in relation to the HS values of temporal series. The annual and seasonal distribution of significant wave heights in the spatial domain shows that the zone of highest incidence, with higher values, is located at the external portion of the continental shelf, above the isobath of $25 \mathrm{~m}$, with an average of $1.96 \mathrm{~m}$ and maximum values above $2 \mathrm{~m}$ for a Hs with ENE directional pattern. In seasonal analyses, during the summer months, the waves are the highest on this coast. In the seasonal and inter-annual analyses, 2010 and 2013 were considered the years of greater agitation and frequency of severe events in the region. The study corroborates for both, knowledge of the waves patterns and the possibility to assess the waves as an energy resource in this sector of the Northeastern coast.
\end{abstract}

Keywords. Surface waves, time series, modeling, seasonality, percentile, RN. 


\section{Introdução}

As ondas oceânicas são de grande interesse para qualquer projeto de engenharia que venham a ser instalados em áreas costeiras, sejam eles próximos da costa ou distantes da costa (Teena et al., 2012). O conhecimento das características das ondas do mar é necessário para prever desastres costeiros e inundações, assim como, o conhecimento das mudanças nas condições das ondas próximas à costa torna-se essencial para o desenvolvimento de medidas de adaptações apropriadas para as construções e obras de engenharia que estão ou virão a ser instaladas (Dreier et al., 2015).

o impacto que uma determinada onda pode causar em uma região costeira depende das grandezas que caracterizam o clima de onda incidente, como por exemplo, altura, comprimento, direção e tempo de duração do mesmo (Chowdhury \& Dhawan, 2016).

Por esta e outras razões, estudar os eventos extremos de ondas tem sido cada vez mais impulsionado pelas ciências ambientais devido às diversas aplicações que estas podem oferecer (Martucci et al., 2010), resultando em ações de prevenção aos danos que venham ocorrer e construir sistemas de mitigação que podem ser usados para combater os efeitos devastadores causados, por exemplo, por ondas como tsunamis, ressacas de mar e outros desastres naturais (Chowdhury \& Dhawan, 2016).

Dessa forma, quando se pretende projetar estruturas oceânicas seguras, é essencial levar em consideração nos estudos às ondas extremas. A análise destas ondas ao largo é a primeira etapa no design de estruturas costeiras para a seleção de alturas de ondas do projeto (Goda et al., 2010).

A estimativa dos valores de projeto contribui para o nível de proteção em escala de investimento. Assim, compreender as condições extremas de onda é de interesse não só da comunidade científica, mas também para o interesse econômico (Teena et al., 2012). Muitos autores nas últimas décadas têm estudado e discutido a análise de valores extremos para ondas, entre eles: Mathiesen et al. (1994), Ferreira \& Guedes Soares (1998), Van Gelder et al. (2000),
Coles (2001), Singh (2006), Roscoe et al. (2010), Martucci et al. (2010), Izaguirre et al. (2011), Caires (2011).

Para Martucci et al. (2010) analisar os valores médios a partir de uma série temporal é de fundamental importância para avaliar uma tendência ao longo de um intervalo de tempo. As amostras dos extremos de séries temporais servem para determinar a probabilidade de ocorrência de eventos extremos e períodos de retorno. Dependendo do método e do critério estatístico da amostragem dos extremos (a partir de uma série temporal), diferentes resultados podem ser obtidos aplicando a mesma análise estatística às diferentes amostras.

$\mathrm{Na}$ costa brasileira a maior parte dos estudos relacionados às análises estatísticas dos extremos de ondas está concentrada na região Sudeste e Sul do país, sendo alguns trabalhos merecedores de destaque, como Parente et al. (2001), Pinho (2003), Cardoso Júnior (2004), Campos (2009), Candella \& Souza (2013), Silva (2013), Guimarães et al. (2014), Campos (2014) entre outros. Boa parte desses autores estudou as ondas da região da Bacia de Campos devido a importância que esta região oferece para a economia brasileira, como a exploração de petróleo em mar, cuja grande parte das atividades é extremamente dependente das condições de mar (Campos et al., 2010). Na região Sul do país, devido aos fortes fenômenos atmosféricos ocorrentes neste setor, como por exemplo, eventos associados aos ciclones e anticiclones extratropicais, e anticiclones do Atlântico Sul, que provocaram ondas acima de $6 \mathrm{~m}$ (Candella \& Souza, 2013), vem sendo amplamente estudada nas últimas décadas.

No Nordeste brasileiro, os estudos têm ganhado cada vez mais importância devido aos aspectos morfodinâmico das praias, vulnerabilidade costeira (Malmann et al., 2014a, 2014b; Costa et al., 2014; Vicente Júnior \& Pereira, 2017) e o conhecimento do clima das ondas para o aproveitamento de energia de ondas (Carvalho, 2010; Farias \& Souza, 2012; Spindola et al. 2016; Spindola \& Araújo, 2017). A caracterização do clima de ondas relacionados ao risco de inundações e erosão da linha de costa do litoral nordestino também têm recebido 
destaque (Scudelari et al., 2016).

Os eventos extremos já vêm resultando em perdas socioeconômicas do setor petrolífero, relacionados à exposição dos dutos submersos (Busman et al., 2014). Também são cada vez mais frequentes, como consequências da erosão costeira, os danos às obras de urbanização nas cidades litorâneas do Rio Grande do Norte (RN) (Busman et al., 2016).

Apesar dos esforços, ainda há muito para se conhecer sobre a climatologia das ondas no litoral do RN, seja através de medições in situ, seja por meio de modelos numéricos de propagação das ondas. O uso de modelos numéricos têm sido uma importante ferramenta para estimação do clima de ondas na asuência de dados in situ a longo prazo.

Atualmente, o Brasil com seus esforços e recursos criou um sistema de monitoramento costeiro pelo Centro de Previsão de Tempo e Estudos Climáticos (CPTEC), do Instituto Nacional de Pesquisas Espaciais (INPE), para toda a costa brasileira, o SIMCos: Coastal Forescasting and Monitoring Coastal System (Sistema de Previsão e Monitoramento Costeiro). O SIMCos gera informações sobre altura significativa, altura potencial, período e direção de ondas que atingem 61 pontos distintos do litoral do brasileiro, foi calibrado com base na reanálise da climatologia das ondas dos útimos 30 anos (1979-2010) do Climate Forecast System Reanalysis (CFSR), do National Centers for Environemnt Prediction (NCEP). Desta forma, o SIMCos prevê (através de simulações) e monitora, variáveis que podem afetar as atividades costeiras (por exemplo, ondas) em diversos pontos da costa.

Contudo, outras bases de dados e sistemas de modelagem numérica têm sido usados para propagar o clima de ondas no $\mathrm{RN}$ em diversas condições, como por exemplo Scudelari et al. (2007), Góis et al. (2009), Fortes et al. (2011), Almeida et al. (2014), Gomes (2017), entre outros. Scudelari et al. (2007) aplicaram inicialmente os modelos REFDIF (Kirby \& Dalrymple, 1994) e SWAN (Booij et al., 1999) para modelar diferentes condições incidentes do litoral de Galinhos e Guamaré (RN). Góis et al. (2009) estudaram a circulação hidrodinâmica da zona costeira frontal a Galinhos e Guamaré por meio do módulo 2DH do modelo hidrodinâmico SisBAHIA (Rosman, 2006). Fortes et al. (2011) aplicaram o modelo SWAN ao litoral setentrional do RN e compararam com medições in situ. Almeida et al. (2014) utilizaram o sistema de monitoramento costeiro, SMC-Brasil, para avaliação do clima de ondas da praia de Ponta Negra (RN). Gomes (2017) executou o modelo SWAN na simulação numérica da incidência de ondas marítimas em praias arenosas de Natal/RN.

Assim, o objetivo deste trabalho é contribuir para o conhecimento do clima de ondas disponível para a costa norte do $\mathrm{RN}$, a partir de bases de dados de simulações numéricas do modelo SWAN para o período de 2010 a 2015, calibrado e validado com dados in situ de várias campanhas realizadas na área entre os anos de 2010 a 2012.

Para tanto, é efetuada uma análise estatística e avaliação da distribuição espacial das alturas significativas das ondas ao longo de uma série temporal entre 2010 a 2015, com análise anual, sazonal e mensal dos padrões de altura significativa (Hs) e direções médias (DIR) em todo o domínio espacial. Além disso, o estudo efetuou uma classificação dos eventos severos de ondas a partir da aplicação da técnica dos percentis, e avaliação dos eventos mais frequentes e sua distribuição ao longo dos meses.

\section{2 Área, materiais e métodos}

\section{1 Localização da área}

Aárea de estudo abrange aproximadamente $270 \mathrm{~km}^{2}$ de área e está situada no litoral setentrional do Rio Grande do Norte (Fig. 1), assinalada por plataforma extensa, rasa e mista, e foi classificado por Vital et al. (2008) em três zonas com características distintas.

Conforme Vital etal. (2008), a porção interna da plataforma continental adjacente é limitada pela isóbata de $15 \mathrm{~m}$, de relevo suave e extensos campos de dunas longitudinais paralelos à costa; na porção média ou intermediária da plataforma, entre os limites das isóbatas de 15 e $25 \mathrm{~m}$ predominam dunas transversais à costa; a porção mais externa da plataforma dá-se a 


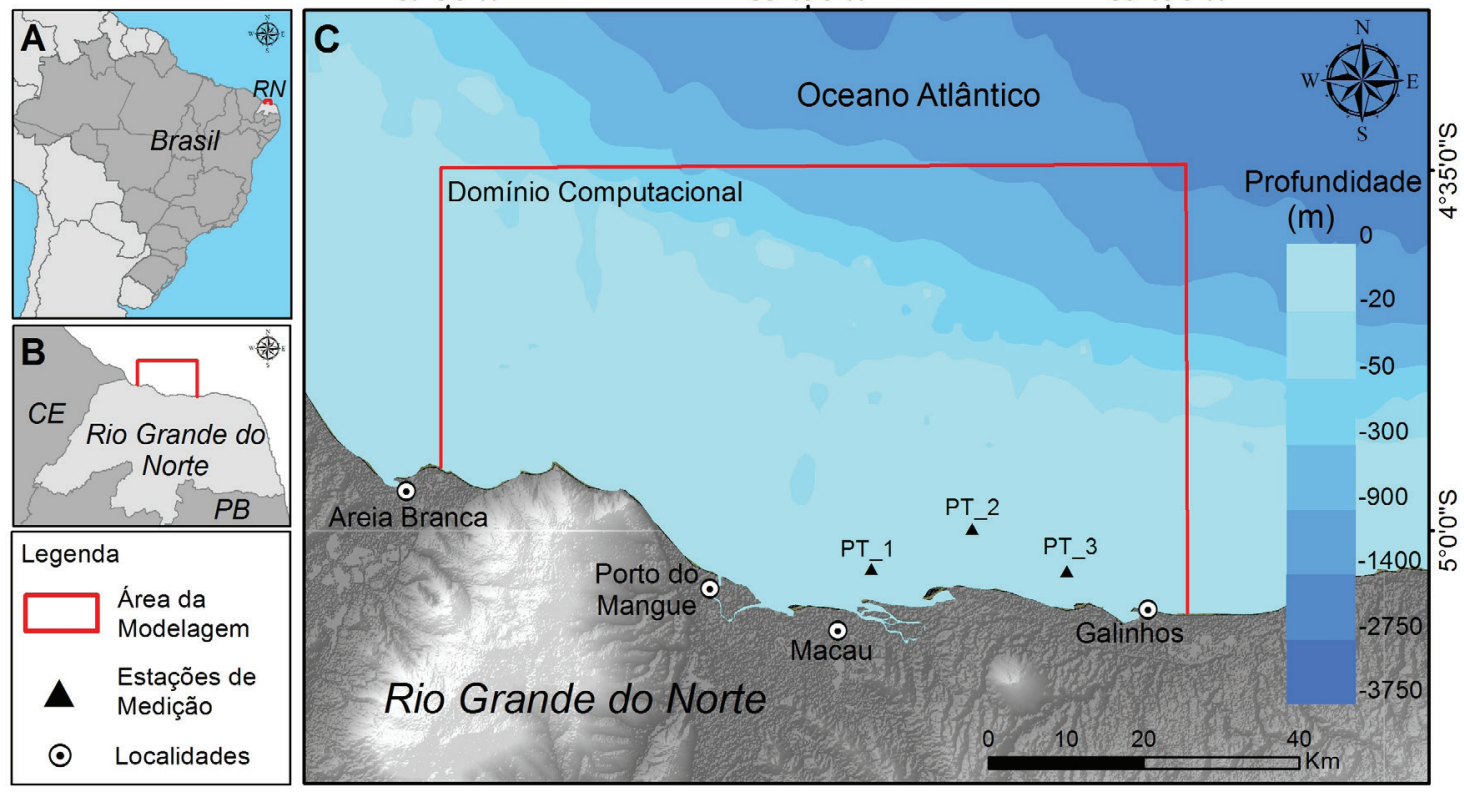

Figura 1. Mapa de localização da área de estudo. A) Localização da área de estudo no Brasil. B) Localização da área de estudo no estado do Rio Grande do Norte. C) Área de estudo destacando o domínio computacional. Base de dados: Modelo digital de elevação extraído do SRTM (USGS, 2016a), com a batimetria da Plataforma Continental Brasileira (CPRM, 2017).

Figure 1. Map of study area. A) Location of the study area in Brazil; B) Location of the study area in Rio Grande do Norte; C) Area of study contrast the computational domain. Data base: Digital elevation model extracted from SRTM (USGS, 2016), and the bathymetry of the Brazilian Continental Shelf (CPRM, 2017).

partir da isóbata de $25 \mathrm{~m}$ até a quebra do talude continental (Fig. 2).

A região apresenta acentuada variabilidade climática interanual, particularmente na precipitação, com alguns anos extremamente secos e outros chuvosos. Está sob a influência dos Anticiclones Subtropicais do Atlântico Sul (ASAS) e do Atlântico Norte (ASAN) e do cavado equatorial, cujas variações sazonais de intensidade e posicionamento determinam o clima na região (Cavalcanti et al., 2009). Os ventos de baixos níveis associados aos sistemas de pressão são os alísios de sudeste, na borda norte do ASAS, e de nordeste, na borda sul do ASAN, os quais apresentam velocidades médias de $6,7 \mathrm{~cm} / \mathrm{s}$, com amplitude entre $2,6-13,4 \mathrm{~cm} / \mathrm{s}$ (Cavalcanti et al., 2009).

No eixo do cavado equatorial está a ZCIT (Zona de Convergência Intertropical), cujas variações em posição e intensidade estão diretamente relacionadas às alterações nas posições e intensidades do ASAS e do ASAN de modo que a ZCIT no Atlântico está na região de convergência dos ventos alísios de nordeste e sudeste, apresentando movimentos ascendentes, baixas pressões, nebulosidade e chuvas abundantes, e segue as regiões onde a TSM (Temperatura da Superfície do Mar) é mais elevada. Esses são os principais fatores dinâmicos que determinam a precipitação sazonal da região (Cavalcanti et al., 2009).

As principais correntes marítimas que margeiam a costa do RN são a Corrente do Brasil, que acompanha o litoral do RN ao Rio Grande do Sul, direção norte-sul, e a Corrente Equatorial que vai do RN ao Amapá, com direção leste-oeste (Rosso, 2007). Embora, estudos hidrodinâmicos sistemáticos sejam ausentes na plataforma continental norte do RN, dados de Matos \& Amaro (2011) registram localmente correntes de altas velocidades, da ordem de $300 \mathrm{~cm} / \mathrm{s}$ nos meses que compõem o verão e, $270 \mathrm{~cm} / \mathrm{s}$ no período de inverno. 


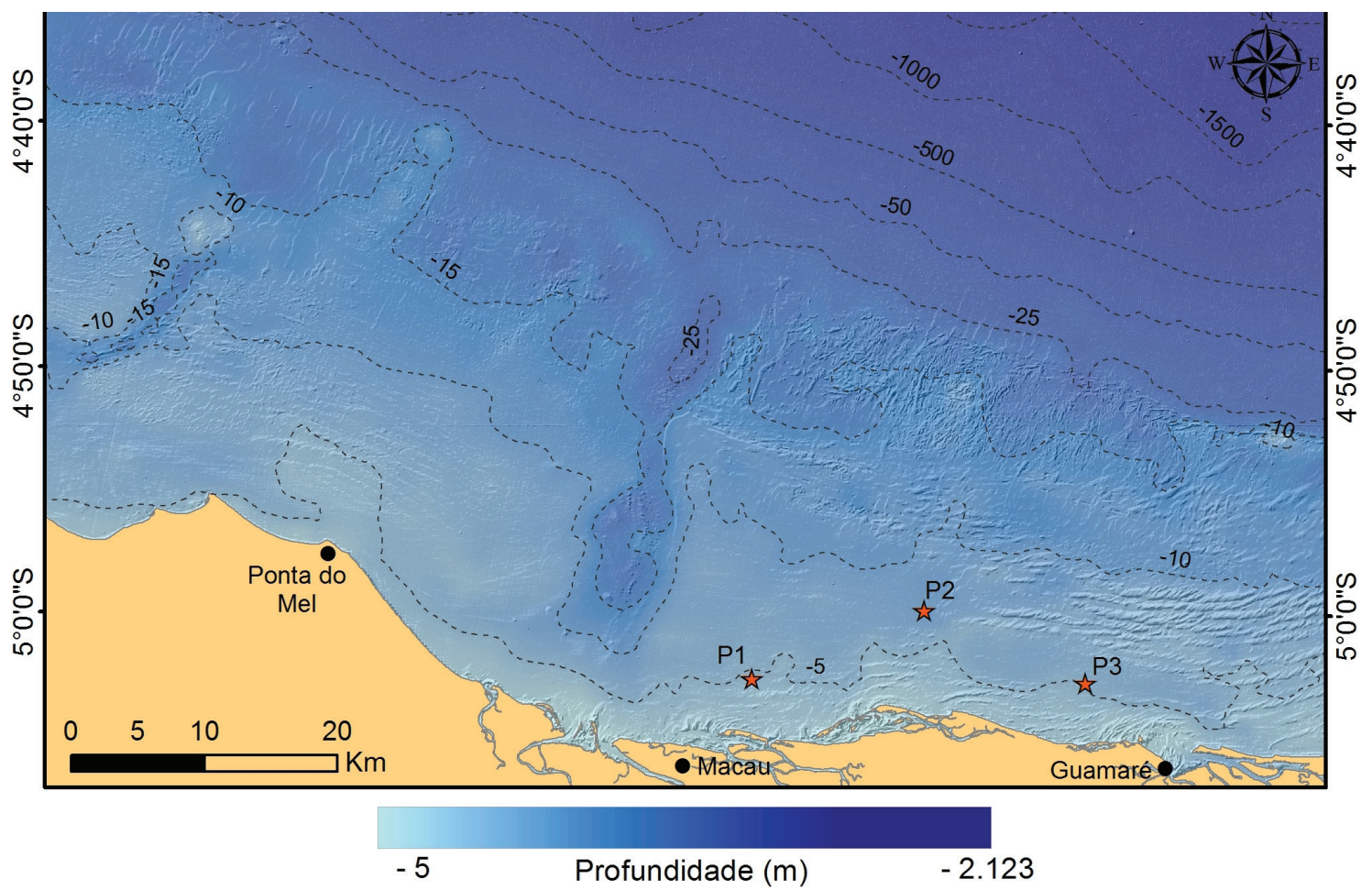

Figura 2. Mapa batimétrico da região usado para o modelo computacional com as isolinhas. Imagem do satélite Landsat OLI de 2016 (USGS, 2016b) com filtro direcional $45^{\circ}$ na banda 8, com sobreposição da batimetria da carta náutica n. 720 (DHN, 2015).

Figure 2. Bathymetric map of the region used for the computational model with contours. Landsat Satellite Image OLI 2016 (USGS, 2016b) with directional filter $45^{\circ}$ in the band 8, with overlapping of the bathymetry of the nautical chart $n$. 720.

A maré local é semidiurna, com duas preamares e duas baixa-mares, onde o nível médio $\left(Z_{0}\right)$ estabelecido é $139 \mathrm{~cm}$ acima do nível de redução com médias de preamares de sizígia de $234 \mathrm{~cm}$ acima do nível de redução, média de preamares de quadratura de $221 \mathrm{~cm}$, média de baixa-mares de sizígia de $43 \mathrm{~cm}$ abaixo do nível de redução e média das baixa-mares de quadratura de $56 \mathrm{~cm}$ (DHN, 2018).

\subsection{Materiais e métodos}

As características das ondas foram obtidas a partir da hindcasting numérica para um período de cinco anos (2010-2015) usando o modelo SWAN (Simulating WAves Nearshore) (Booij et al., 1999), calibrado para o litoral norte do RN por Matos et al. (2013). O SWAN foi operacionalizado pela ferramenta de interface SOPRO/LNEC (Santos et al., 2005, Fortes et al., 2011).

Em Matos et al. (2013) foi determinado e definido todos os parâmetros necessários para a calibração do modelo SWAN: batimetria, condições de fronteira ao largo, ventos, marés, domínio computacional (Tab. 3) e os fenômenos físicos considerados nos cálculos, quais sejam: (i) Malha externa: a refração, o empolamento e a rebentação por influência do fundo e do excesso de declividade (whitecapping), a difração e interações onda-onda quádruplas (quadruplet wave-wave interactions); (ii) $\mathrm{Na}$ malha intermediária e interna: a refração,o empolamento e a rebentação por influência do fundo e do excesso de declividade (whitecapping), as interações entre tríades de ondas (triad wavewave interactions), a geração de harmônicas (frequency shifting) e a difração.

A calibração foi baseada em duas campanhas de campo nos três pontos (PT_1, PT_2 e PT_3): dezembro de 2010 e fevereiro de 2011, com dados obtidos simultaneamente nos dois pontos. Os resultados apresentados por Matos et al. (2013) foram de modo geral satisfatórios em termos de propagação e evolução das ondas, apresentando similaridades entre os valores medidos em relação aos simulados. As maiores diferenças encontradas foram relacionadas às direções de onda, devido 
os campos de ventos introduzidos no domínio computacional serem constantes. Em Matos et al. (2017) foram introduzidos campos de ventos corrigidos, cujos resultados foram considerados neste trabalho.

\subsubsection{Dados de ondas}

Os dados de ondas usados para calibração e validação do modelo numérico (Matos et al., 2013), foram obtidos a partir de várias campanhas de campo (Tab. 1), com o uso de dois perfiladores acústicos de medição de ondas e correntes: o AWAC - Acustic Wave and Current Meter, e o AQUADOPP PROFILER. Ambos os instrumentos são de mesmo fabricante, NORTEK AS, e foram configurados para obter os parâmetros de ondas: altura significativa, período médio e direção média. Os parâmetros de correntes não fizeram parte deste trabalho.

As datas das campanhas foram intercaladas em vários períodos devidos a acessibilidade da área: novembro e dezembro de 2010; janeiro e fevereiro de 2011; abril, maio e agosto de 2012 .

Os dados foram processados pelo software STORM do mesmo fabricante, NORTEK AS, para obter os parâmetros da onda (altura significativa, altura máxima, período de pico, período médio, direção de pico e direção média). O software utiliza como método e algoritmo padrão para o processamento, o MLMST (Método Máxima Verossimilhança com Rastreamento de Superfície). Este método é o mais indicado para o processamento de séries de medições de onda com AWAC AST (Kahma et al., 2005; Pedersen et al., 2007). Por este método, é obtido um significativo conjunto de estimativas de onda: estimativa da altura da onda significativa ( $\mathrm{Hs}, \mathrm{m})$; período médio (Tmed, s) baseado nos momentos de espectros de energia; direção média (Dir, ${ }^{\circ}$ ) que corresponde à direção de energia ou à direção média. A ponderação de energia é aplicada aos coeficientes de Fourier.

$\mathrm{Na}$ tabela 2 é apresentado um resumo estatístico geral das ondas usadas para validação do modelo SWAN, contendo valores: máximo, média, mínimo e frequência dos parâmetros de ondas em cada estação costeira: PT_1, PT_2 e PT_3. Com relação aos dados in situ, os parâmetros de ondas da estação PT_2 são superiores as demais estações, devido a esta estação encontrar-se localizada mais distante da costa e em maior profundidade.

Tabela 1. Localização de cada instrumento de medição e características de coleta.

Table 1. Location of measuring instruments and collection settings.

\begin{tabular}{|c|c|c|c|c|c|c|}
\hline \multirow{2}{*}{ Estação } & \multirow{2}{*}{ Instrumento } & \multirow{2}{*}{$\begin{array}{c}\text { Prof. } \\
\text { (m) }\end{array}$} & \multicolumn{2}{|c|}{ Localização } & \multirow{2}{*}{$\begin{array}{l}\text { Média } \\
\text { (s) }\end{array}$} & \multirow{2}{*}{$\begin{array}{l}\text { Freq. } \\
\text { (MHz) }\end{array}$} \\
\hline & & & Latitude & Longitude & & \\
\hline PT_1 & AQUADOPP & 3 & $-5^{\circ} 2 ’ 37,76 ” S$ & $36^{\circ} 35^{\prime} 38,64^{\prime \prime} \mathrm{W}$ & 60 & 1,0 \\
\hline PT_2 & AWAC & 8 & $-4^{\circ} 59^{\prime} 50,49^{\prime \prime} \mathrm{S}$ & $36^{\circ} 28^{\prime} 37,57^{\prime \prime} \mathrm{W}$ & 60 & 1,0 \\
\hline PT_3 & AQUADOPP & 5 & $-5^{\circ} 2^{\prime} 467,74^{\prime \prime} \mathrm{S}$ & $36^{\circ} 22^{\prime} 3,82^{\prime \prime} \mathrm{W}$ & 60 & 1,0 \\
\hline
\end{tabular}

Tabela 2. Estatística descritiva dos dados in situ e suas diferenças de validação do modelo. Table 2. Descriptive statistics of in situ data and their model validation differences.

\begin{tabular}{|c|c|c|c|c|c|c|c|c|c|}
\hline & \multicolumn{3}{|c|}{ PT_1 } & \multicolumn{3}{|c|}{ PT_2 } & \multicolumn{3}{|c|}{ PT_3 } \\
\hline & Hs (m) & Tmed (s) & DIR $\left(^{\circ}\right)$ & Hs (m) & Tmed (s) & $\operatorname{DIR}\left({ }^{\circ}\right)$ & Hs (m) & Tmed (s) & $\operatorname{DIR}\left({ }^{\circ}\right)$ \\
\hline Máximo & 1,25 & 7,48 & 359,2 & 2,04 & 8,18 & 359,9 & 1,73 & 4,86 & 69 \\
\hline Média & 0,74 & 5,06 & 185,6 & 1,15 & 4,70 & 80,6 & 1,38 & 3,59 & 61 \\
\hline Mínimo & 0,39 & 3,19 & 0,17 & 0,67 & 3,37 & 0,15 & 0,92 & 3,15 & 55 \\
\hline Freq. & 0,80 & $3-4$ & $50-90$ & 0,90 & $4-5$ & $0,3-15$ & 1,10 & 3,6 & 62 \\
\hline
\end{tabular}




\subsubsection{Modelo SWAN}

Neste estudo, o modelo SWAN foi usado para determinação do clima de ondas, com resoluções espaciais e temporais de $1 \mathrm{~km}$ e $6 \mathrm{~h}$, respectivamente. Utilizou-se dados batimétricos da carta náutica número 720, obtida a partir da Diretoria de Hidrografia e Navegação (DHN), da Marinha do Brasil com resolução espacial de $1 \mathrm{~km}$ como dado de entrada para o modelo na representação do fundo marinho. As medições de ondas próximas da costa foram usadas para a verificação/calibração do modelo (Matos et al., 2017). Para os cálculos com o modelo SWAN, foram utilizadas três malhas encaixadas, cujas dimensões e localização são apresentadas na tabela 3. Cabe ressaltar que a necessidade de três malhas está relacionada com o melhor desempenho do modelo numérico face à utilização de apenas uma malha, quer no que se refere à qualidade da previsão, quer ao tempo necessário ao cálculo.

Tabela 3. Característica do domínio computacional e limite das malhas.

Table 3. Computational domain characteristic and grid limit.

\begin{tabular}{lcccc}
\hline \multicolumn{1}{c}{ Estação } & X inicial & Y inicial & Dimensões $(\mathbf{k m})$ & Resolução (x,y) \\
\hline M1 - Externa & 710000 & 9436000 & $100 \times 62$ & $(1000,1000)$ \\
M2 - Intermediária & 750000 & 9440000 & $50 \times 20$ & $(500,250)$ \\
M3 - Interna & 753000 & 9440000 & $30 \times 10$ & $(100,50)$ \\
\hline
\end{tabular}

Assim, as características da agitação ao largo constituem a condição de fronteira na malha global (Externa), em um ou dois dos lados dessa fronteira, dependendo da direção de incidência da agitação. Os resultados da malha global fornecem as condições de fronteira para a $2^{\mathrm{a}}$ malha (Intermediária), e os resultados desta malha fornecem as condições de fronteira para a $3^{a}$ malha (Interna).

As simulações no modelo SWAN foram realizadas na versão $40.72 \mathrm{AB}$, no modo bidimensional, estacionário de terceira geração para cada um dos processos, e nas formulações padrões. Considerando que há dois tipos de aproximação que descrevem os mecanismos de transferência da energia do vento para a superfície livre do mar: um considera um crescimento da energia da onda linear no tempo (Komen et al., 1984), e outro considera um crescimento exponencial (Ris, 1997). Ambos, o crescimento linear e o exponencial de entrada do vento, foram incluídos no modelo.

O modelo SWAN converte os valores de entrada do campo de ventos medidos a $10 \mathrm{~m}$ de elevação $U_{10}$ navelocidade de atrito $u_{*}$ (Holthuijsen, 2007). Assim, os campos de ventos introduzidos no domínio computacional, e referentes ao período de análise do estudo, são caracterizados pelos valores de velocidade $(\mathrm{m} / \mathrm{s})$ e direção do vento $(\theta)$. Tais valores foram adquiridos, através da base de dados da estação meteorológica de Macau - A317 (INMET, 2013). Devido à estação encontrar-se localizada em terra, foi necessário fazer uma correção da velocidade dos ventos medidos, de forma a representar as condições dos ventos de mar. Esta correção foi baseada em Resio \& Vincent (1977) e Rosman (2006). O detalhamento da correção encontra-se em Matos et al. (2017). Portanto, o vento assumiu valores típicos de zona marítima, variáveis ao longo do tempo.

A dissipação devido à rebentação induzida pelo fundo é tratada por Battjes \& Janssen (1978) com a formulação espectral com $a=1,0$ atrito de fundo é modelado usando a formulação JONSWAP (Hasselmann et al., 1973) com coeficiente de atrito $C=0,015 \mathrm{~m}^{2} \mathrm{~s}^{-3}$ em condições wind sea e Komen et al. (1984) para a formulação whitcapping ou excesso de declividade é aplicado $C_{d s}=2,36 \times 10^{-5}$, e um coeficiente de rebentação constante igual a $0,65 \mathrm{~m}^{2} \mathrm{~s}^{-3}$.

As interações não-lineares entre grupos de três ondas são descritas pelo modelo SWAN através da aproximação de Lumped Triad (Eldeberky, 1996). No modelo SWAN existem duas formulações distintas para 
descrever as interações de grupos de quatro ondas: a formulação DIA (Discrete Interaction Approximation) proposta por Hasselmann et al (1985) e a formulação WRT (Webb-Resio-Tracy) de Van Vledder (2006). Para a condução do trabalhofoiusadoàformulaçãoDIA para descrever as interações não-lineares de grupos de quatro ondas. A definição destas configurações padrões em regime estacionário resulta, principalmente, em simulação mais econômica em termos de tempo, considerando que o processamento foi realizado continuamente ao longo de todos os meses.

As condições de agitação ao largo introduzidas foram as do modelo de $3^{a}$ geração WAVEWATCH III, da bóia localizada na costa nordeste do Brasil nas coordenadas: 787460,84 E/9666781,81 S, zona UTM-24 Sul, Datum WGS1984. A bóia está situada na profundidade de 3.350,0 m, distante da costa aproximadamente $230 \mathrm{~km}$. As informações fornecidas pela mesma correspondem aos seguintes parâmetros de onda: Hs (altura significativa), $T_{p}$ (período médio) e DIR (direção média para a frequência de pico), obtidas a cada ciclo de 6 horas. As ondas ao largo possuem alturas máximas de 3,53 m, médias de 1,89 m, com períodos máximos de 14,8s e períodos médios de $7,3 \mathrm{~s}$, sendo as direções predominantes de $80^{\circ}$ e $90^{\circ}$ (Leste-Nordeste).

A partir destes valores integrais, $\mathrm{Hs}$ e $T_{p}$ definiu-se um espectro JONSWAP $\operatorname{com} \gamma=3,3$ para representar a dependência de frequência. O espectro direcional foi discretizado em 23 intervalos de baixa frequência, variando entre 0,04 e 1,0 Hz, com distribuição logarítmica. A discretização em direção abrange $360^{\circ}$, dividida em 90 intervalos.

\subsubsection{Técnica dos percentis para eventos extremos de ondas}

Para a quantificação dos eventos severos de ondas, utilizou-se a técnica dos percentis (Wilks, 2006) para as séries temporais de Hs de 2010 - 2015 obtidas a partir do modelo SWAN. A escolha desta técnica é baseada em Martucci et al. (2010), que sugere a escolha de um limiar, no caso um percentil (P) fixo, do conjunto de dados de altura de onda ordenada, garantindo assim que o valor limite depende diretamente da série temporal dos dados. A tabela 4 mostra a ordem crescente dos percentis de $\mathrm{P}=80,90$, 95 e 99\% para as distribuições da série temporal das alturas significativas, Hs (P significa o limite do percentil adotado para o conjunto de dados de cada estação costeira).

De acordo com a tabela 4, a evolução dos percentis aponta um clima de onda mais severo no percentil $P_{0,99}$ para cada estação (Tab. 2), correspondente a valores acima de: 1,08 m, 1,34 e 1,13 m, respectivamente, PT_1, PT_2 e PT_3, e por isso, este percentil foi definido como o valor limite para estimar os valores extremos de Hs nas três estações costeiras.

\section{Resultados e discussões}

\subsection{Análises estatísticas}

A partir dos dados simulados no modelo SWAN, observou-se que os maiores valores de Hs que chegaram ao largo da plataforma continental setentrional do RN, entre os anos de 2010 e 2015, superaram os 1,08, 1,34 e 1,13 m (limiares do percentil 99), em cada estação costeira com valores máximos de 1,69, 2,05 e 1,48 m (Tab. 5).

Tabela 4. Série temporal de alturas significativas, relativo aos percentis para cada estação costeira.

Table 4. Time series of significant heights, percentiles for each coast station.

\begin{tabular}{ccccc}
\hline Estação & $\mathbf{P}_{\mathbf{0 , 8 0}}$ & $\mathbf{P}_{\mathbf{0 , 9 0}}$ & $\mathbf{P}_{\mathbf{0 , 9 5}}$ & $\mathbf{P}_{\mathbf{0 , 9}}$ \\
\hline PT_1 & 0,56 & 0,70 & 0,82 & 1,08 \\
PT_2 & 0,77 & 0,89 & 1,02 & 1,34 \\
PT_3 & 0,73 & 0,86 & 0,96 & 1,13 \\
\hline
\end{tabular}


Tabela 5. Estatística descritiva da série temporal (2010-2015) dos parâmetros de ondas: altura significativa, período médio e direções médias.

Table 5. Descriptive statistics of time series (2010-2015) of the wave parameters: significant height, mean period and mean directions.

\begin{tabular}{|c|c|c|c|c|c|c|c|c|c|}
\hline & \multicolumn{3}{|c|}{ PT_1 } & \multicolumn{3}{|c|}{ PT_2 } & \multicolumn{3}{|c|}{ PT_3 } \\
\hline & Hs (m) & Tmed (s) & $\operatorname{DIR}\left(^{\circ}\right)$ & Hs (m) & Tmed (s) & $\operatorname{DIR}\left(^{\circ}\right)$ & Hs (m) & Tmed (s) & $\operatorname{DIR}\left(^{\circ}\right)$ \\
\hline Máximo & 1,69 & 8,4 & 360 & 2,05 & 8,13 & 360 & 1,48 & 6,88 & 360 \\
\hline Média & 0,32 & 5,3 & 35 & 0,53 & 4,63 & 57 & 0,49 & 4,27 & 60 \\
\hline Mínimo & 0,19 & 3,1 & 0,12 & 0,15 & 2,67 & 0,007 & 0,20 & 2,24 & 0,018 \\
\hline Erro Padrão & 0,003 & 0,01 & 0,22 & 0,003 & 0,009 & 0,37 & 0,003 & 0,007 & 0,55 \\
\hline Desvio Padrão & 0,26 & 0,77 & 21 & 0,28 & 0,80 & 34 & 0,25 & 0,65 & 52 \\
\hline
\end{tabular}

A maior parte da série ocorreu em torno de 0,40 e 0,80 m.

As direções máximas que incidem na costa chegam a $360^{\circ}$ (Norte, $\mathrm{N}$ ), as médias variando entre $35^{\circ}$ a $60^{\circ}$ (Nordeste, NE e Leste-Nordeste, ENE), voltando a incidir com direções mínimas de $\mathrm{N}$, de $0^{\circ}$ a $0,12^{\circ}$.

Quanto à distribuição das frequências da série temporal observada, a ocorrência das ondas mais frequentes que atingem a costa varia entre as estações (Fig. 3): no PT_1 as frequências de 0,40 $\mathrm{m}$ representam mais de $60 \%$ das alturas significativas que ocorrem; no PT_2 as alturas entre 0,40 e 0,80 m são as mais frequentes, somando mais $80 \%$ em relação às demais frequências. No PT_3 a densidade de ocorrência de Hs possui uma maior distribuição entre as frequências ao longo da série, variando com os maiores percentuais entre 0,20 a 1,00 m. Estes resultados são similares às frequências de distribuição global das alturas significativas encontrados por Soares et al. (2001) e Chu et al. (2010) para as regiões de baixas latitudes, entre $0^{\circ}-20^{\circ} \mathrm{S}$ (Young, 1999). Essa regularidade de frequências se dá em função dos ventos de leste soprarem durante todo o ano nesta costa (Salcedo-Castro et al., 2018).

Em termos de períodos médios, destacaram-se as frequências de 5 a $7 \mathrm{~s}$ no PT_1, de 4 a 6 s no PT_2, e no PT_3, os períodos médios das ondas possuem maior distribuição de ocorrência ao longo da série temporal, variando entre 3,5 a $6 \mathrm{~s}$, indicando um estado de agitação marítima constante, dado pelos valores apresentados.

A distribuição de frequência das direções médias da série temporal mostra que a região é dominada pelas ondas de NE e ENE. As direções são relativamente constantes durante todo o ano. Isso indica que a geometria da plataforma continental setentrional impede que os sistemas provenientes do Hemisfério Norte penetrem com maior intensidade no Hemisfério Sul.

\subsection{Distribuição espacial anual e sazonal das alturas significativas das ondas}

O clima de ondas foi calculado para cada ponto da malha computacional do modelo, de modo a cobrir $270 \mathrm{~km}^{2}$ de área do litoral setentrional do RN. A figura 4 mostra a média anual dos cinco anos de análise da distribuição espacial das alturas significativa de ondas. De acordo com a figura 3, a altura significativa da onda é mais elevada nas profundidades acima de $25 \mathrm{~m}$, zona conhecida como plataforma continental externa. Ao longo do ano, nesta zona, a altura significativa da onda possui uma média de 1,96 m e valores máximos acima de 3,5 m, verificados nas zonas mais profundas, acima de $2.000 \mathrm{~m}$ de profundidade.

$\mathrm{Na}$ zona mista, ou plataforma continental intermediária, entre as isóbatas de 15 a $25 \mathrm{~m}$, a Hs varia de 1,0 m a 1,2 m. Uma característica marcante nesta zona é a presença de uma longa faixa de beachrocks, com mais de $50 \mathrm{~km}$ de extensão, que faz com que as ondas ao largo ao alcançarem esta zona intermediária tendam a se submeter aos efeitos de refração, empolamento e difração devido às variações espaciais na profundidade, ou seja, a interação com o fundo marinho mais raso provoca a refração das ondas quando da transição de um ambiente para outro, fenômeno este reproduzido pelo modelo SWAN (Ris, 1997). 

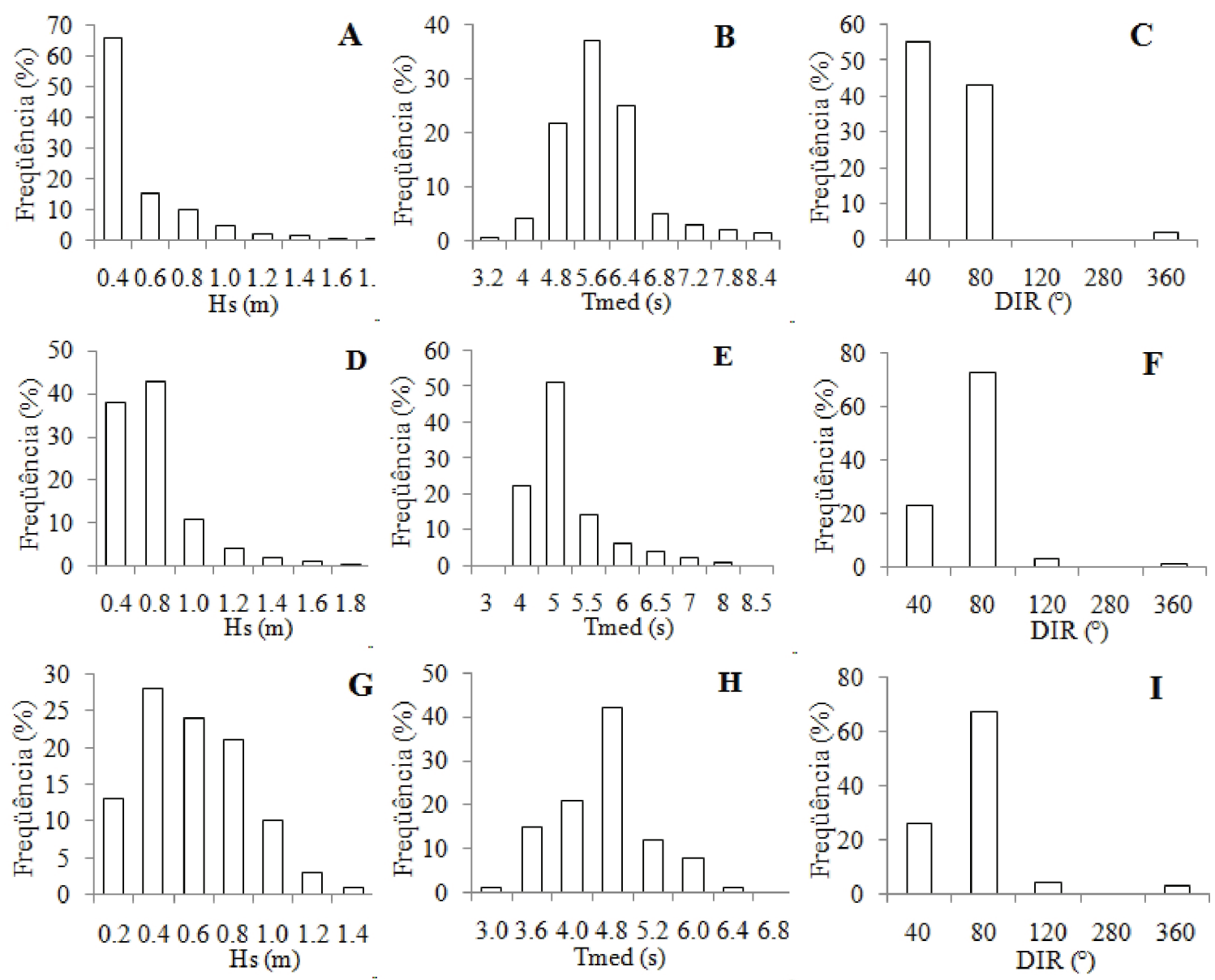

Figura 3. Frequência de ocorrência do clima de ondas da série temporal simulada. A) Altura significativa do PT_1; B) Período médio do PT_1; C) Direção média do PT_1; D) Altura significativa do PT_2; E) Período médio do PT_2; F) Direção média do PT_2; G) Altura significativa do PT_3; H) Período médio do PT_3; I) Direção média do PT_3.

Figure 3. Frequency of occurrence of the wave climate of the simulated time series. A) Significant heights of PT_1; B) Mean period of $P T_{-}$; ; C) Mean direction of $P T_{-} 1$; D) Significant heights of $P T_{-}$2; E) Mean period of PT_2; F) Mean direction of PT_2; G) Significant heights of PT_3; H) Mean period of PT_3; I) Mean direction of PT_3.
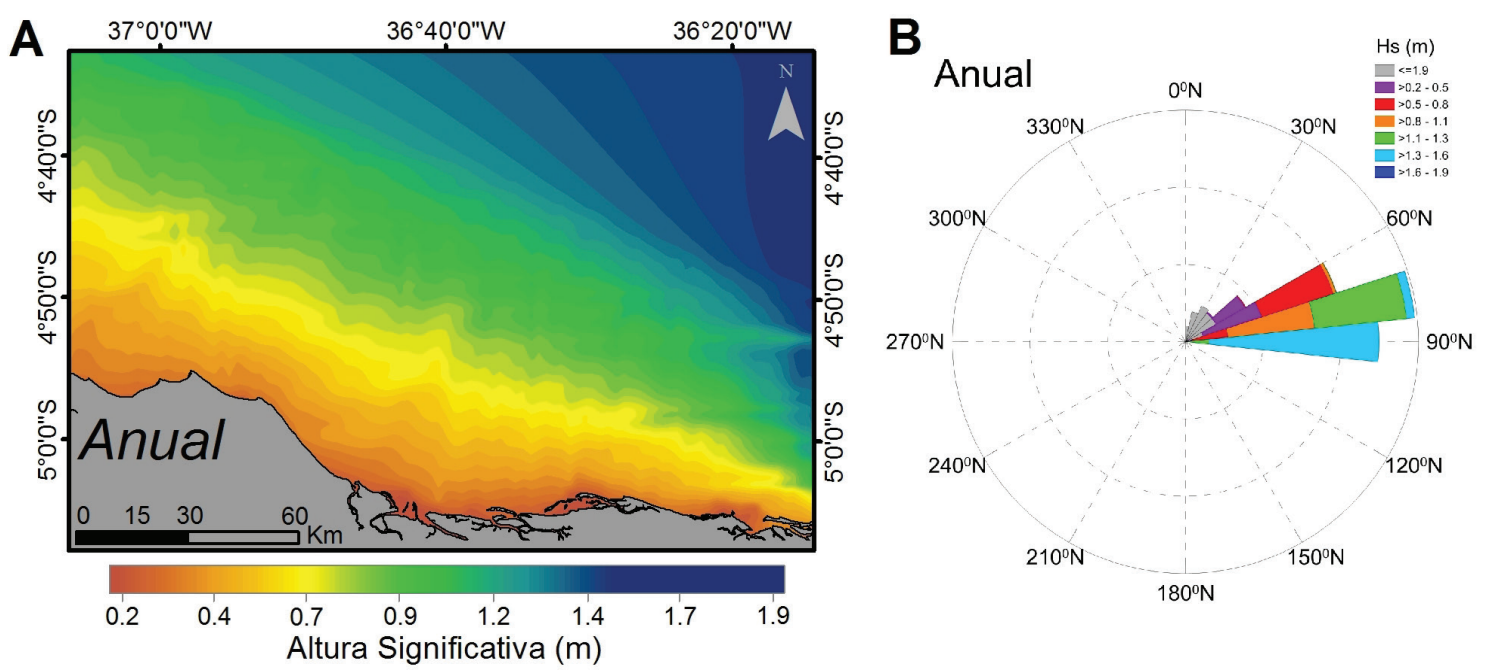

Figura 4. Distribuição espacial da média anual. A) Espacialização da altura significativa da onda ao longo da costa; B) Distribuição das direções médias.

Figure 4. Spatial distribution of significant height annual average. A) Significant wave height spacing along the RN coast; B) Distribution of annual average direction. 
Após a zona dos beachrocks, a presença dos campos de dunas transversais no fundo marinho da zona mista, causa o efeito de difração na propagação da onda, modificando assim a direção de propagação da mesma e reduzindo sua altura no sentido da praia. Essas características são muito mais evidentes durante os meses de verão e de outono (Fig. 5), correspondentes aos meses de dezembro a abril (Fig. 7) quando as ondas predominam de NNE (Norte-Nordeste) na região.

Na zona costeira, ou plataforma continental interna, limitada pela isóbata de até $15 \mathrm{~m}$, as alturas de ondas chegam muito reduzidas, com média de variação de 0,20 a 0,80 m de Hs. Desta forma, caracteriza-se uma incidência anual de agitação marítima muito baixa em relação às demais zonas da plataforma continental. Quando as ondas atingem a zona de praia, tendem a refratar ainda mais devido o efeito causado pelas variações das baixas profundidades, em função da presença de extensos campos de
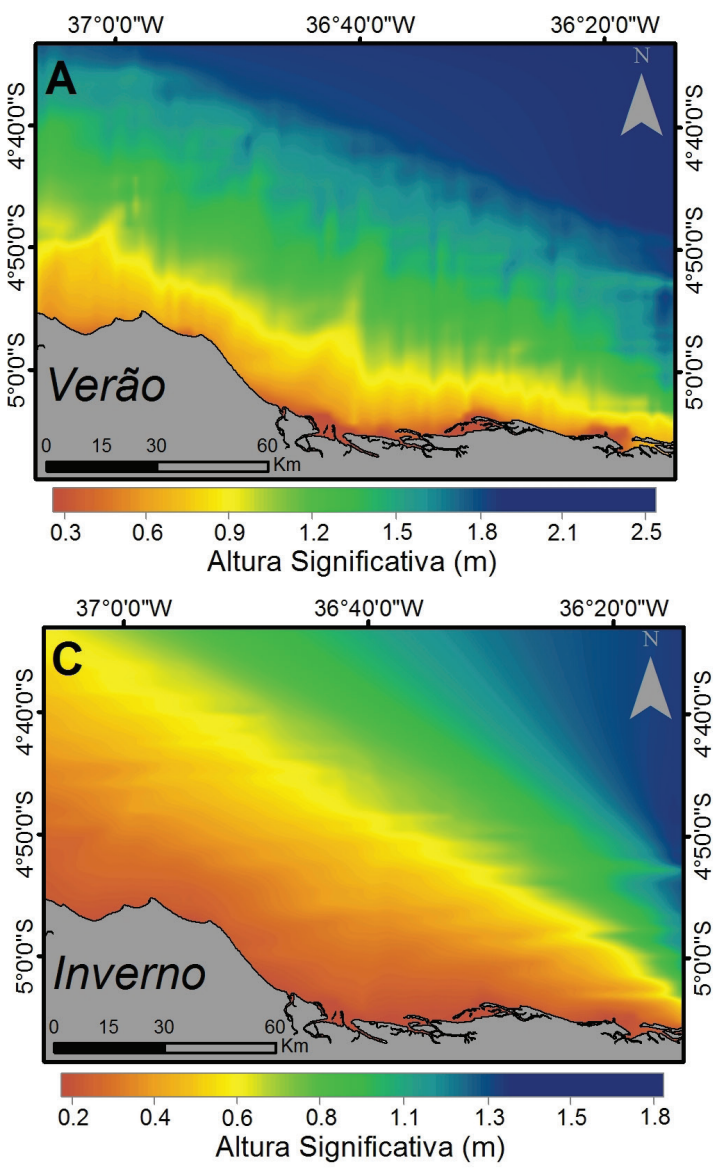

dunas longitudinais submersos paralelos à costa presentes nesta região.

Além das alturas significativas, é importante observar o comportamento dos padrões direcionais da onda. Conforme a figura 4, a direção de propagação anual da onda prevalece de ENE, embora as ondas mais altas se propaguem de NNE.

Com o objetivo de atribuir uma distribuição espacial da variabilidade sazonal ao longo da série temporal analisada, foram efetuados os cálculos de Hs e DIR para cada estação (Fig. 5 e 6). Assim, conforme a figura 5 foi identificado que as maiores alturas de ondas se propagam durante 0 verão, atingindo alturas mais elevadas acima de 2,0 m no domínio geográfico. No outono há uma redução das alturas, alcançando 1,9 m e atinge os valores mais baixos durante o inverno, 1,76 m. Subsequentemente, na primavera, as alturas significativas voltam a crescer para alcançar os maiores valores no verão.
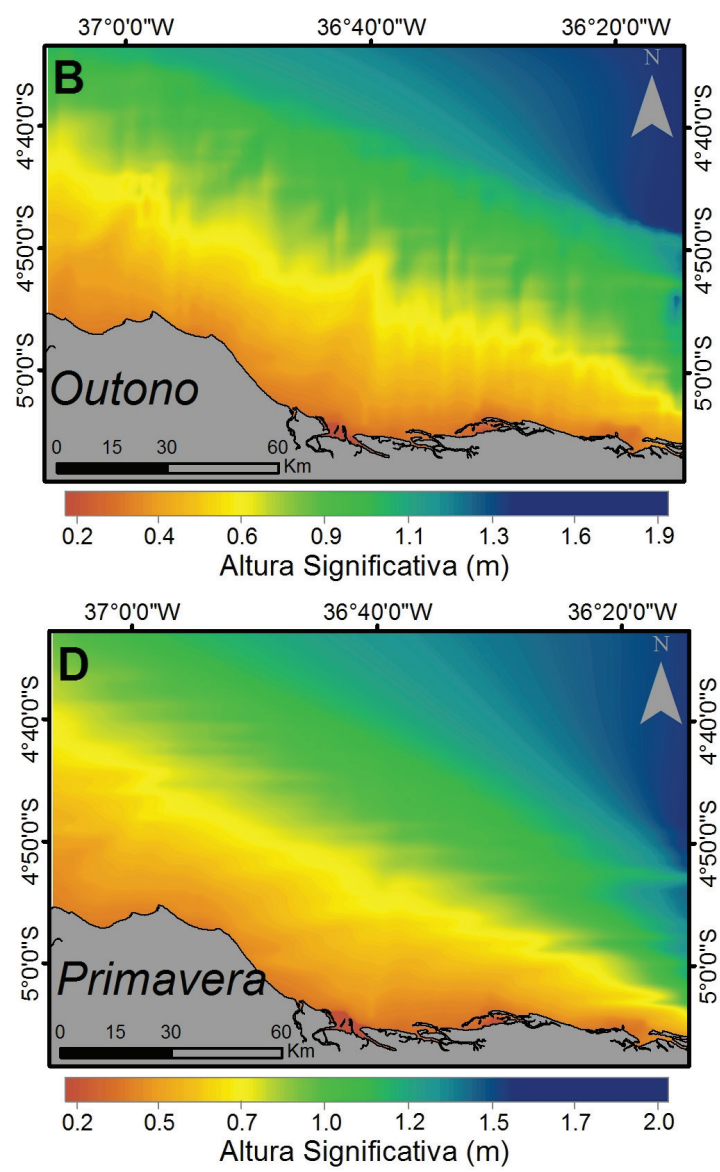

Figura 5. Distribuição espacial sazonal da agitação marítima na costa do Rio Grande do Norte. A) Altura significativa no verão; B) Altura significativa no outono; C) Altura significativa no inverno; D) Altura significativa na primavera.

Figure 5. Seasonal spatial distribution of sea agitation on coast of Rio Grande do Norte. A) Significant height in summer; B) Significant height in autumn; C) Significant height in winter; Significant height in spring. 


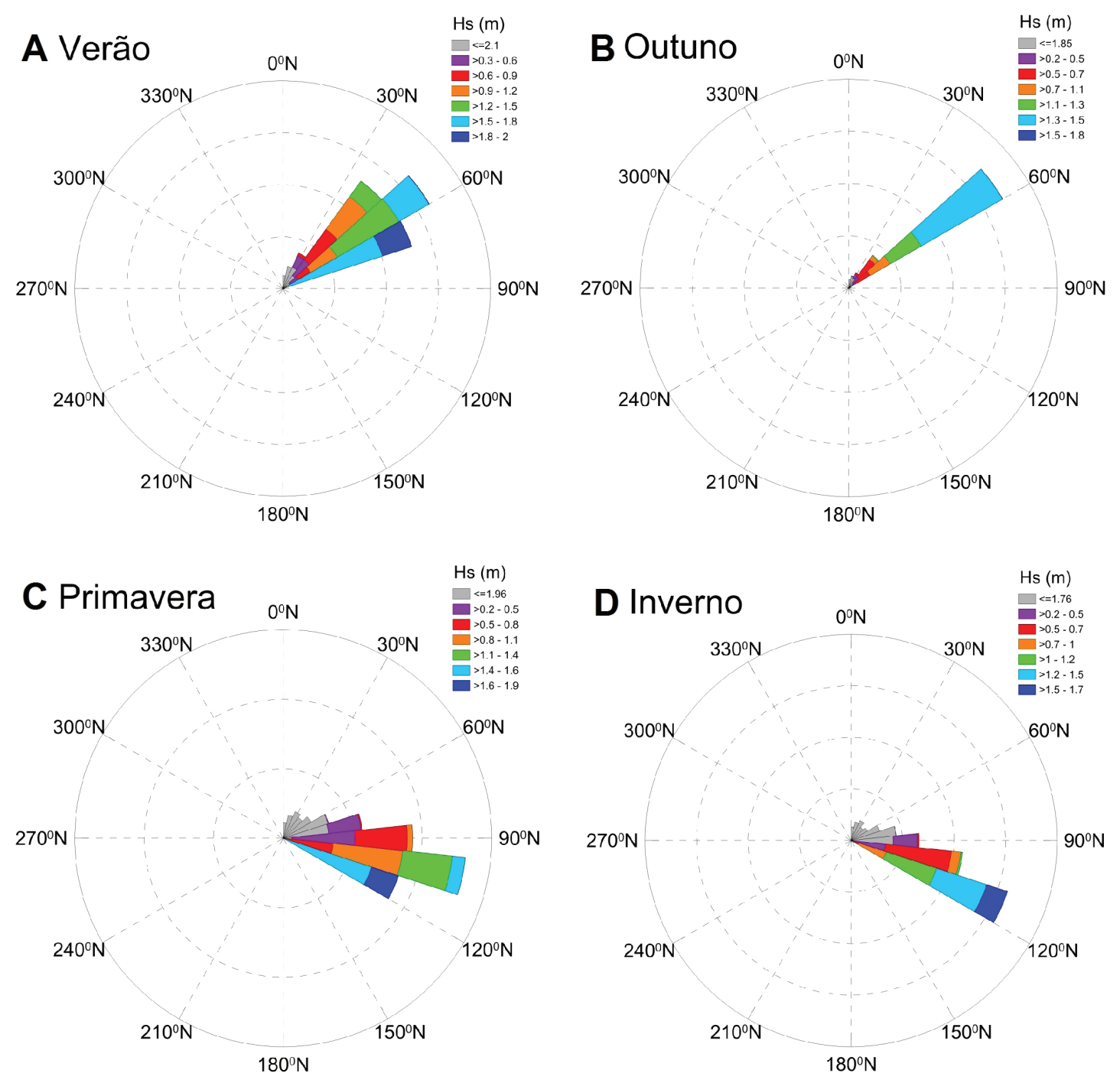

Figura 6. Distribuição sazonal dos padrões direcionais da onda. A) Direções médias de verão; B) Direções médias de outono; C) Direções médias de inverno; D) Direções médias de primavera.

Figure 6. Seasonal distribution of directional wave patterns. A) Means summer direction; B) Means autumn directions; C) Means winter directions; D) Means spring directions.

Nas características da distribuição sazonal, a figura 5 mostra as variações espaciais em relação às zonas da plataforma continental. Durante o verão e outono é muito mais evidente estas variações espaciais e a interação com o fundo marinho.

Nos meses que compõe o verão: dezembro, janeiro e fevereiro (DJF), as alturas significativas são predominantemente de NNE (Fig. 6), entretanto, apesar de ser a estação com ondas mais elevadas, estes padrões direcionais estão associados à média das ondas mais frequentes, que variam entre $\mathrm{Hs}$ de 1,6 e 1,8 m.

Durante o outono: março, abril e maio (MAM) predominam padrões direcionais de NE e ENE, sendo as ondas mais frequentes as que apresentam alturas significativas de 1,6 e 1,8 m.

Nos meses que caracterizam o inverno: junho, julho e agosto (JJA), quando as alturas das ondas reduzem significativamente para médias de 0,7 m, com maior de frequência de 0,2 a 0,8 $m$, nota-se nos padrões direcionais que as ondas incidentes, propagam-se principalmente de ESE (Leste-Sudeste), quando os ventos de leste e os alísios de sudestes são mais frequentes e intensos nesse período.

$\mathrm{Na}$ primavera encontram-se dois padrões direcionais predominantes: de ESE, cuja frequência de ondas corresponde as com alturas de 1,8 m e, uma segunda predominância de $E$, com frequência de ondas de 0,4 m.

Devido a essa porção do litoral ser voltado 
para o HN (Hemisfério Norte), Silva (2013) explica que alturas de ondas significativas mais elevadas no verão nessa região, estão associadas aos ventos alísios provenientes do HN, sendo estes mais intensos nesse período que os provenientes do HS (Hemisfério Sul), implicando assim, em ondas mais fortes durante esta estação. Silva (2013) também obteve valores similares de Hs de 2,5 m para a costa Nordeste.

Semedo et al. (2011) também realizaram estudos similares e explicam que o outro fator responsável pelas alturas de ondas significativas elevadas são os períodos de pico que alcançam essa região, oriundos dos ciclones tropicais e extratropicais que são mais intensos no HN durante o verão, e tornam-se mais elevados devido ao posicionamento da ZCIT mais ao sul nessa época do ano (Cavalcanti et al., 2009).

\subsection{Distribuição espacial mensal das alturas significativas das ondas}

A variação mensal da distribuição espacial das alturas das ondas (Fig. 7) mostra que as Hs mais elevadas são encontradas nas zonas de águas mais profundas nos meses de dezembro, janeiro e fevereiro, coincidentemente a estação do verão, atingindo alturas de 2,1 m em dezembro e fevereiro. De janeiro a abril, observa-se que a média de Hs de 0,8 m alcança a zona costeira, nas proximidades da isóbata de $5 \mathrm{~m}$. Os valores mais baixos foram observados nos meses de julho, agosto e setembro. Nestes meses a média de Hs de 0,70 m, encontra-se próxima das profundidades de $25 \mathrm{~m}$, sendo as áreas abaixo desta isóbata até a zona de praia, os valores são sempre muito baixos, com médias de Hs de 0,2 a $0,4 \mathrm{~m}$.

Ao longos dos meses, verifica-se claramente a evoluação das ondas que atingem a costa norte do $\mathrm{RN}$, de janeiro a abril são predominantes de NNE e NE. A partir do mês de maio, a costa é atingida por ondas de E, incidindo posteriormente para padrões de ESE e SE nos meses de junho, julho e agosto. Em seguida, nos meses de setembro, outubro e novembro as ondas tornam-se a propagar de ESE e E, até volta a incidir de NE e ENE no mês de dezembro.

Considerando as zonas da plataforma continental, verifica-se que a mudança direcional de acordo com as variações de profundidades são fortemente percebíveis nos meses de janeiro a abril, quando as ondas ao largo (geradas em profundidades acima de $1.000 \mathrm{~m}$ ) alcançam a zona da plataforma intermediária (limitada a produnfidade de $25 \mathrm{~m}$ ), dissipando e transferindo energia na costa.

Ao largo as ondas se propagam com direções predominantes de ENE, com maior frequência de ondas de $60^{\circ}$ e $65^{\circ}$, ao entrar na zona intermediária há uma mudança predominante de $\mathrm{NE}, 45^{\circ}$. Ao atingir a costa, a maior frequência das ondas varia entre $20^{\circ}$ e $40^{\circ}$, ou seja, de NNE.

A partir dessas análises mensais da distribuição espacial, percebe-se que o clima de ondas segue os padrões de Hs e DIR esperados nessa região, especialmente, porque a dinâmica que controla o clima de ondas no Nordeste do Brasil está associado diretamente com a ZCIT e alguns sistemas meteorológicos que originam ondas provenientes do Hemisfério Norte, como as tempestades tropicais (Silva, 2013), então, ao longo do ano, o clima de ondas responde favoravelmente ao deslocamento da ZCIT.

\subsection{Temporalidade sazonal e mensal nas estações costeiras}

Foram obtidas as séries temporais das características das ondas para as três estações costeiras e foram determinadas as variações sazonais das alturas máxima e média (Fig. 8 e 9). A figura 8 revela que em todos os três pontos, a média mais alta existe no verão, enquanto que o valor mais baixo existe no inverno. Além disso, a média de HS do PT_2 é de 0,80 m, superior às demais estações, sobretudo em relação ao PT_1. Embora as alturas máximas da onda ocorram em menor frequência nos meses de verão e no outono, os valores sazonais também foram considerados. A figura 8 também indica que nas estações do PT_1 e PT_3, as alturas máximas existem no outono, enquanto que no PT_2 a altura máxima da onda é observada no verão e outono, acima de $2 \mathrm{~m}$ de Hs.

As variações mensais dos índices estatísticos do clima de ondas, incluindo a média e a máxima são mostrados na figura 9. 

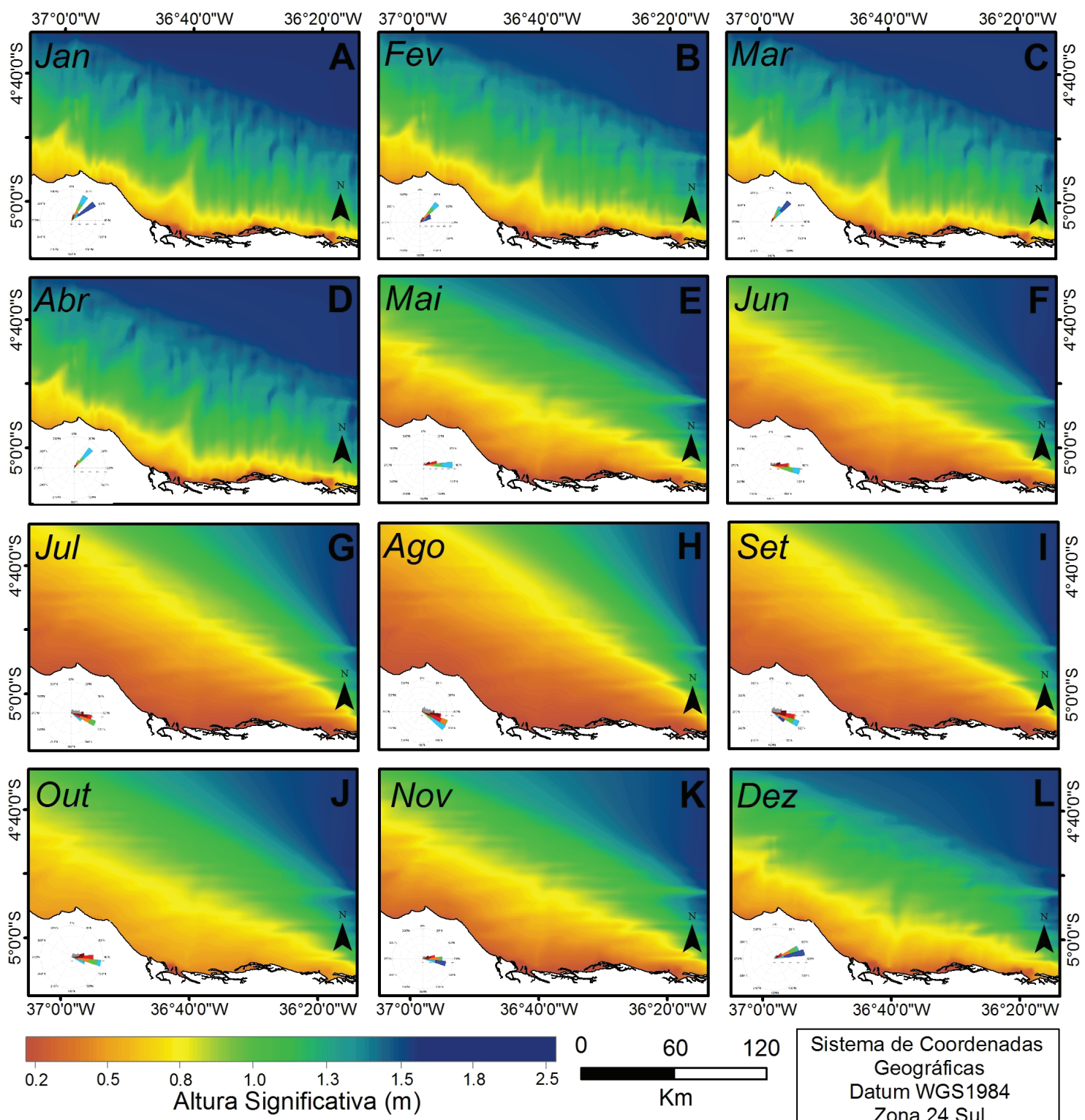

Sistema de Coordenadas
Geográficas
Datum WGS1984
Zona 24 Sul

Figura 7. Distribuição espacial mensal das alturas significativas da onda ao longo da costa do Rio Grande do Norte. A) Altura significativa em janeiro; B) Altura significativa em fevereiro; C) Altura significativa em março; D) Altura significativa em abril; E) Altura significativa em maio; F) Altura significativa em junho; G) Altura significativa em julho; H) Altura significativa em agosto; I) Altura significativa em setembro; J) Altura significativa em outubro; K) Altura significativa em novembro; L) Altura significativa em dezembro.

Figure 7. Monthly spatial distribution of significant directional patterns and heights of the waves. A) Significant height in january; B) Significant height in february; C) Significant height in march; D) Significant height in april; E) Significant height in may; F) Significant height in june; G) Significant height in july; H) Significant height in august: I) Significant height in september; J) Significant height in october; K) Significant height in november; L) Significant height in december.

De acordo com esta figura o PT_2 concentra as maiores alturas, médias e máximas, assim como também, a maior diferença entre os valores máximos de fevereiro a março em relação aos valores mínimos do mês de agosto. Há uma grande variação entre os meses de janeiro a abril com relação ao PT_1 e PT_2 tanto nos valores médios quanto nos valores máximos. Isso ocorre em função do PT_1 encontrar-se muito mais próximo da costa.

\subsection{Variações interanuais do clima de onda nas estações costeiras}

A avaliação da variabilidade interanual do clima de ondas é fundamental para considerar as variações anuais nas alturas e períodos da onda. A análise mostra que há uma diminuição significativa em um período de cinco anos nas três estações, com exceção do PT_2 que apresentou um aumento considerável nos valores máximos 

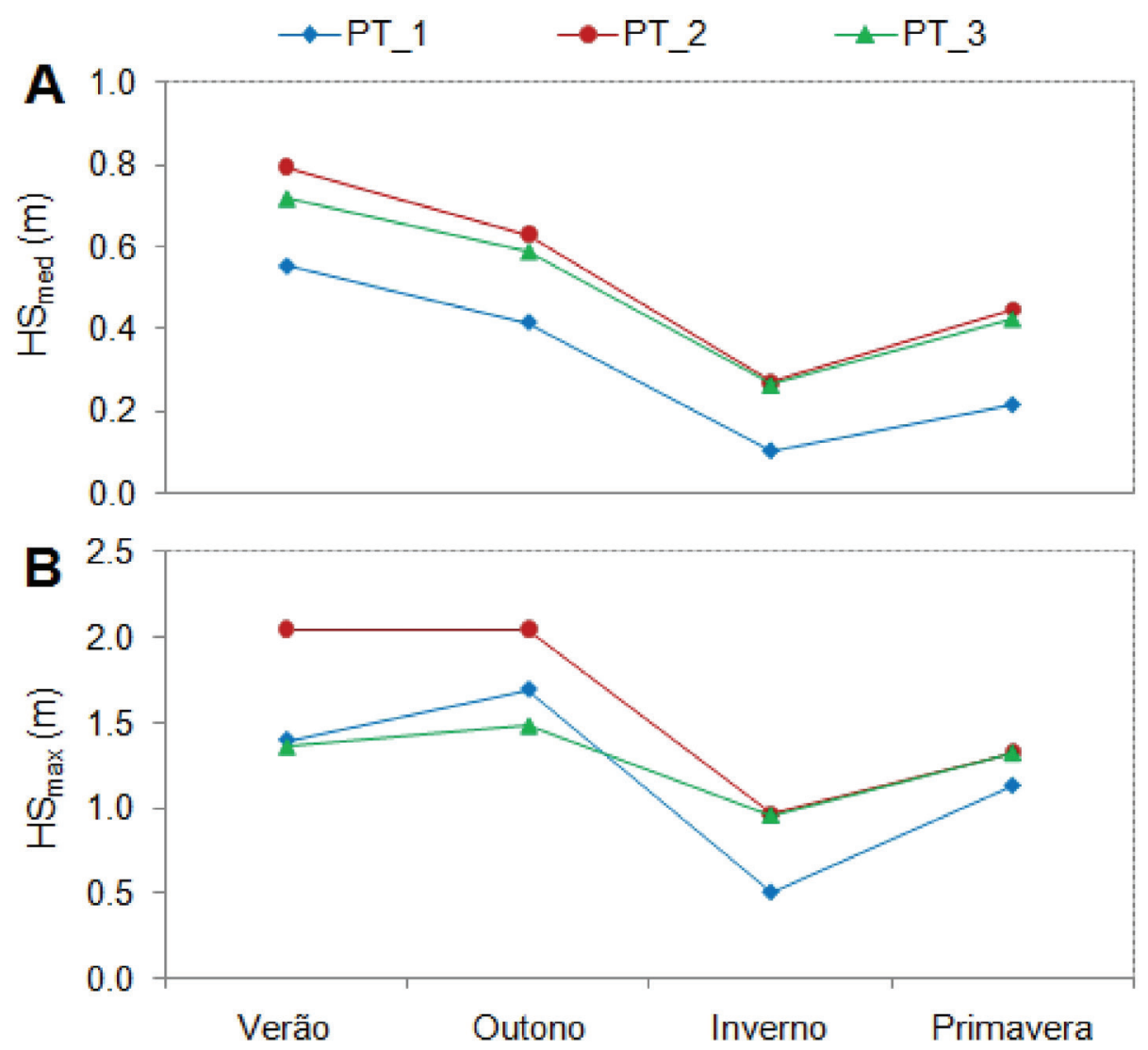

Figura 8. Temporalidade sazonal das alturas de ondas significativas nas estações costeiras. A) Altura significativa média da onda; B) Altura significativa máxima da onda.

Figure 8. Seasonal distribution of wave significant heights in the coastal stations. A) Mean significant wave height; B) Maximum significant wave height.
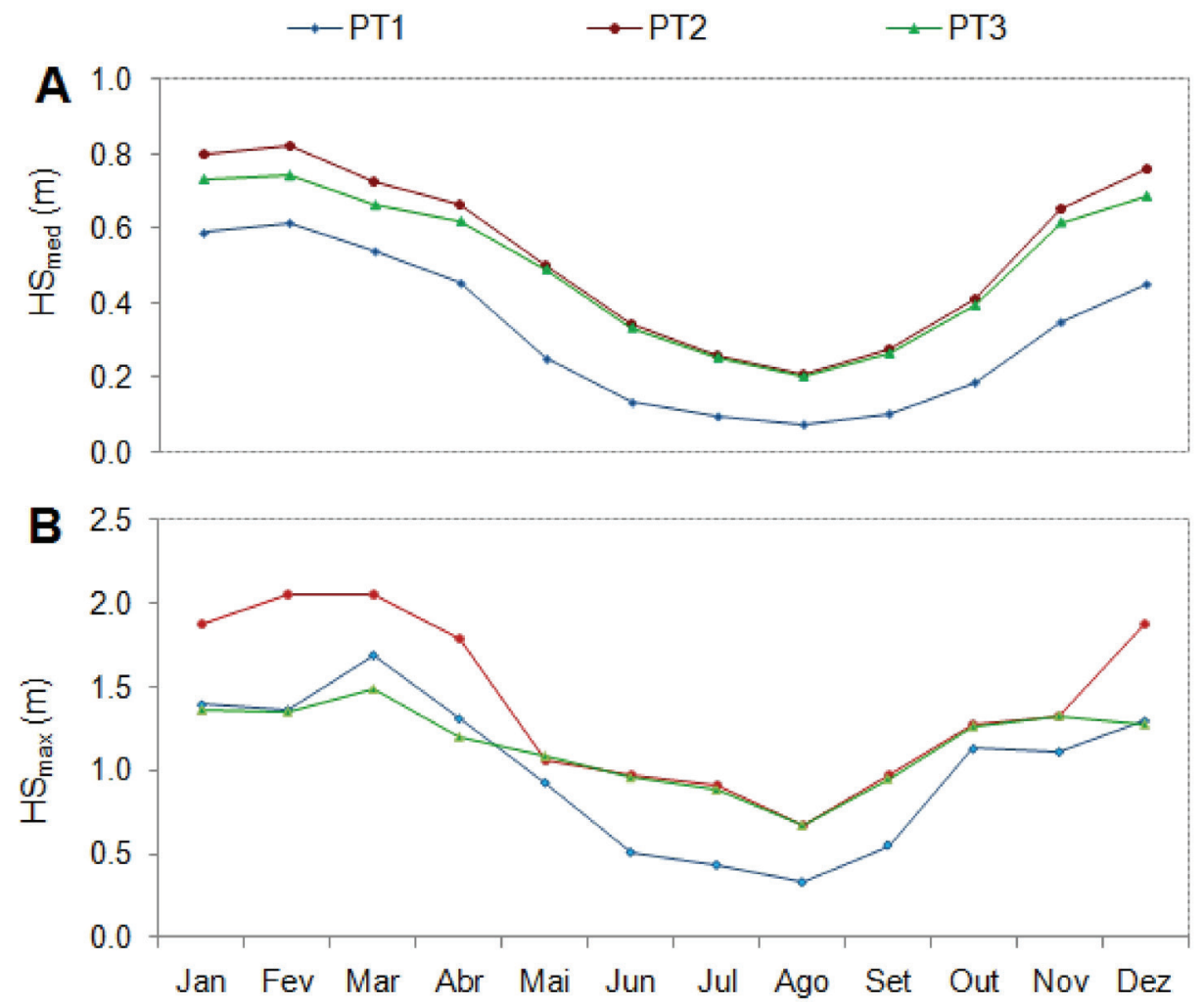

Figura 9. Temporalidade mensal dos valores máximos e médios das alturas de ondas significativas nas estações costeiras. A) Altura significativa média da onda; B) Altura significativa máxima da onda.

Figure 9. Distributions of maximum and average monthly values of significant wave heights in the coastal stations. A) Mean significant wave height; B) Maximum significant wave height. 
de Hs em 2013 (Fig. 10A), acima de 2 m de altura. Nas demais estações observa-se sempre uma tendência à diminuição, com aumento em 2013, porém, não tão brusco quando comparado com o PT_2.

Os períodos médios da onda, Tmed, também mostram uma diminuição significativa em todas as estações (Fig. 10C e 10D), apesar de que os períodos médios não foram tão atenuantes em 2013 como ocorreu com as alturas significativas, porém, mostrou significativamente um aumento nos valores máximos em 2013 no PT_2 e nos valores máximos em 2012 e 2014 para o PT_1 e PT_3. Em relação às características dos valores médios, nota-se sempre uma tendência a diminuição em todas as estações.

\subsection{Composição dos eventos extremos}

Ofocodestaetapaécaracterizareclassificar os eventos de ondas mais severas entre 2010 a 2015. Foi feito primeiramente a quantificação dos eventos, em seguida, determinou-se os padrões de direções desses eventos e, posteriormente, foi classificado os horários de maior concentração dos eventos extremos de alturas significativas, Hs.
Ao aplicar a técnica dos percentis sobre toda a série temporal, extraiu-se para cada estação costeira os valores máximos, todos associados ao percentil acima de $\mathrm{P}_{0,99}$, visto que este demonstrou, conforme a tabela 4, as alturas mais severas para cada estação.

A figura 11 mostra a distribuição temporal dos eventos para cada estação costeira ao longo dos anos de 2010 a 2015. Foram quantificados um total de 91 eventos no PT_1; 88 eventos no PT_2; e, 93 eventos no PT_3, sendo que estes eventos representaram apenas a média de 1\% diante da série temporal diária em cada estação.

Na figura 11 A, referente ao PT_1, verifica-se que os anos de 2010, 2011 e 2013, apresentaram uma média de 26 a 28 eventos mais extremos, no PT_2, a concentração é em 2010 e 2013, com média de 36 a 37 eventos, e no PT_3, ocorreu uma média de 21 a 27 eventos mais extremos. Nas três estações, os últimos dois anos foram muito reduzidos de eventos. Dentre os anos analisados, em 2011, foi o único ano sem registro de eventos acima do percentil $P_{0,99}$ no PT_2. Assim, o ano de 2013, resultou ser o que apresentou os eventos mais extremos.
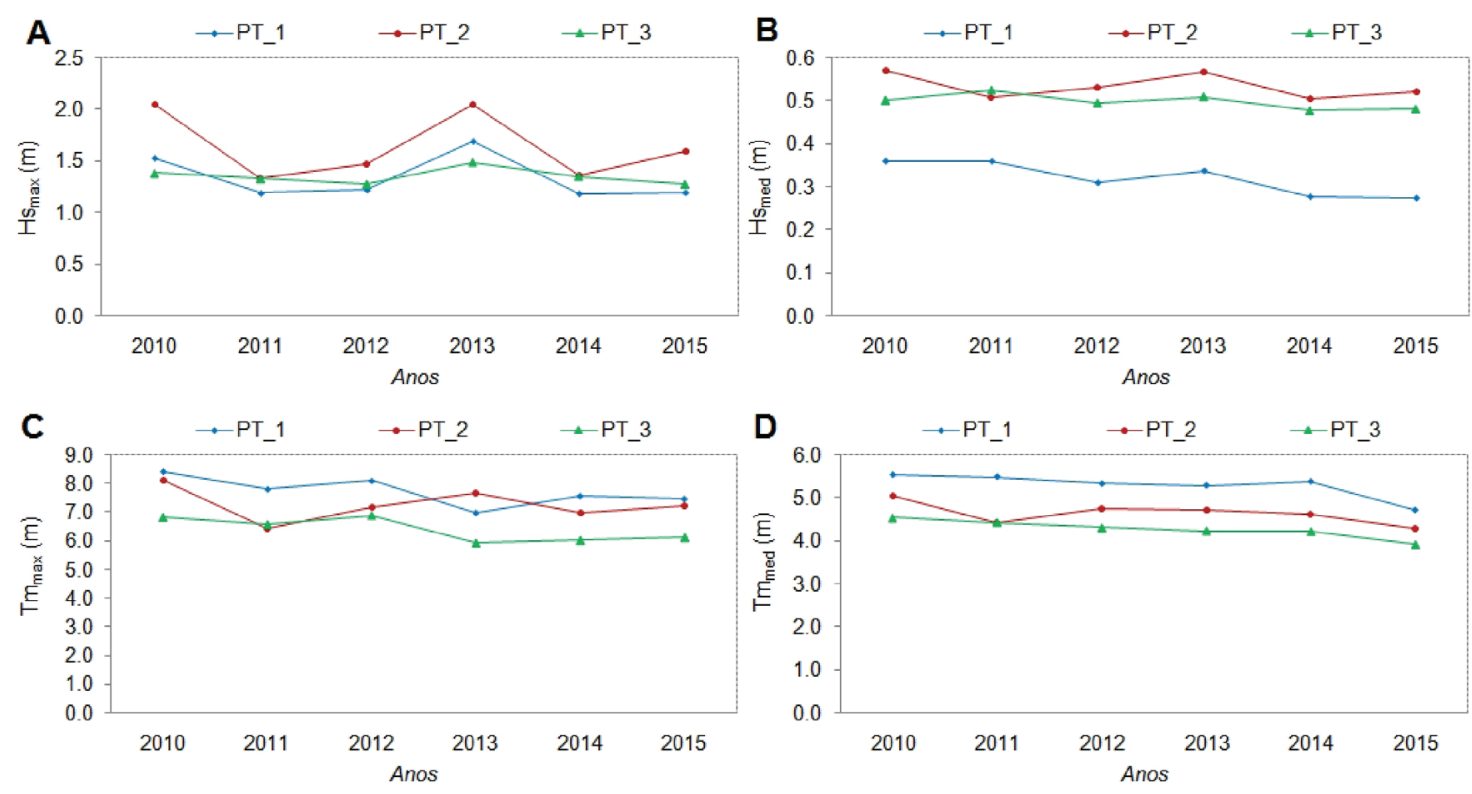

Figura 10. Distribuições interanuais. A) Alturas significativas máximas de onda nas três estações costeiras; B) Alturas significativas médias de onda nas três estações costeiras; C) Período de pico de onda nas três estações costeiras; D) Período médio de onda nas três estações costeiras.

Figure10. Inter-annual distribution. A) Maximum significant wave heights in the three coastal stations; B) Significant mean wave heights at the three coast stations; C) Peak wave period at the three coast stations; D) Average wave period at the three coast stations. 

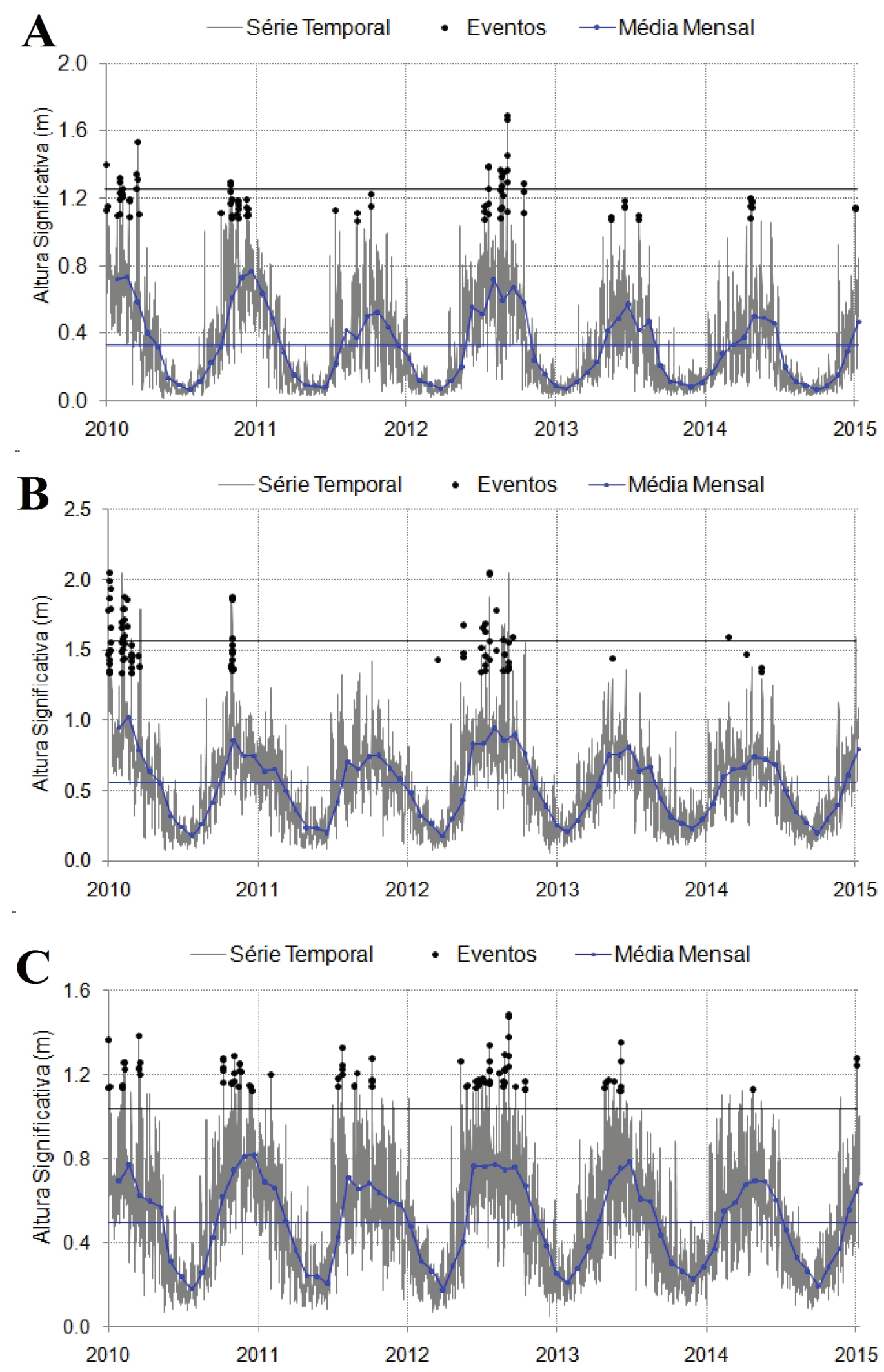

Figura 11. Concentração de eventos de ondas acima do percentil 99 para os valores de HS para as três estações costeiras. Na cor cinza são é a série temporal; a cor azul é a média mensal de cada ano; e, os pontos pretos são os valores extremos para cada parâmetro. A) Estação costeira PT_1; B) Estação costeira PT_2; C) Estação costeira PT_3.

Figure 11. Concentration of events of waves above the 99 percentile values of HS to three coastal stations. In gray are the temporal series; the color blue is the monthly average of each year; and the black dots are the extreme values for each parameter. A) Coastal station PT_1; B) Costal station PT_2; C) Coastal station PT_3. 
Nafigura12,épossívelanalisarafrequências de ocorrência dos eventos e identificar que os eventos mais frequentes estão relacionados com Hs de 1,2, 1,5 e 1,1 m, respectivamente aos PT_1, PT_2 e PT_3. Estes eventos estão associados, predominantemente, aos sistemas provenientes de Norte, entre as direções de NNE nos PT_1 $\left(4^{\circ}\right.$ a $8^{\circ}$ ) e PT_2 $\left(5^{\circ}\right.$ a $\left.15^{\circ}\right)$, e de N, NE e ENE no PT_3 $\left(10^{\circ}\right.$ a $60^{\circ}$ e $\left.360^{\circ}\right)$.

Com o objetivo de analisar o padrão de incidência desses eventos extremos, foram construídos os diagramas direcionais para verificar o comportamento em cada estação. Conforme a figura 13, os eventos possuem padrões de NNE, no PT_1 e PT_2, sendo que no PT_3, há um padrão bimodal, com eventos de $\mathrm{N}$ variando a ENE.

Devido a maior frequência desses eventos ocorrerem nos meses de dezembro, janeiro e fevereiro, isso sugere que é durante o verão que os eventos extremos de ondas ocorrem nessa região.

Para olhar a distribuição dos eventos extremos no tempo, estes foram agrupados mensalmente, como mostrado na figura 14. As barras em azul claro estão relacionadas com os eventos na estação PT_1; o vermelho escuro com o PT_2; e, o verde com os eventos identificados no PT_3.

É possível identificar que a maioria dos eventos ocorre nos meses que compõe o verão (dezembro, janeiro e fevereiro), e parte do outono (março e abril), entretanto, em todos os pontos, o mês de fevereiro concentrou os maiores percentuais, 39, 31 e 28\%, respectivamente. Semedo et al. (2011) e Silva (2013) explicam que as ondas do litoral norte do nordeste brasileiro voltada para o HN, são mais fortes nessa estação e recebe a influência dos ventos alísio do HS, assim como o ASAS, que durante o verão está mais deslocado para leste no Atlântico Sul, intensificando assim o período de pico e as ondas mais severas nessa região.

Diferentemente nas demais estações do ano, verifica-se praticamente que não ocorreu nenhum evento extremo durante o inverno, devido o período transitório das frentes oscilatória se movendo para o Norte, voltando a acumular na primavera (Young, et al., 1999; Semedo et al., 2011).
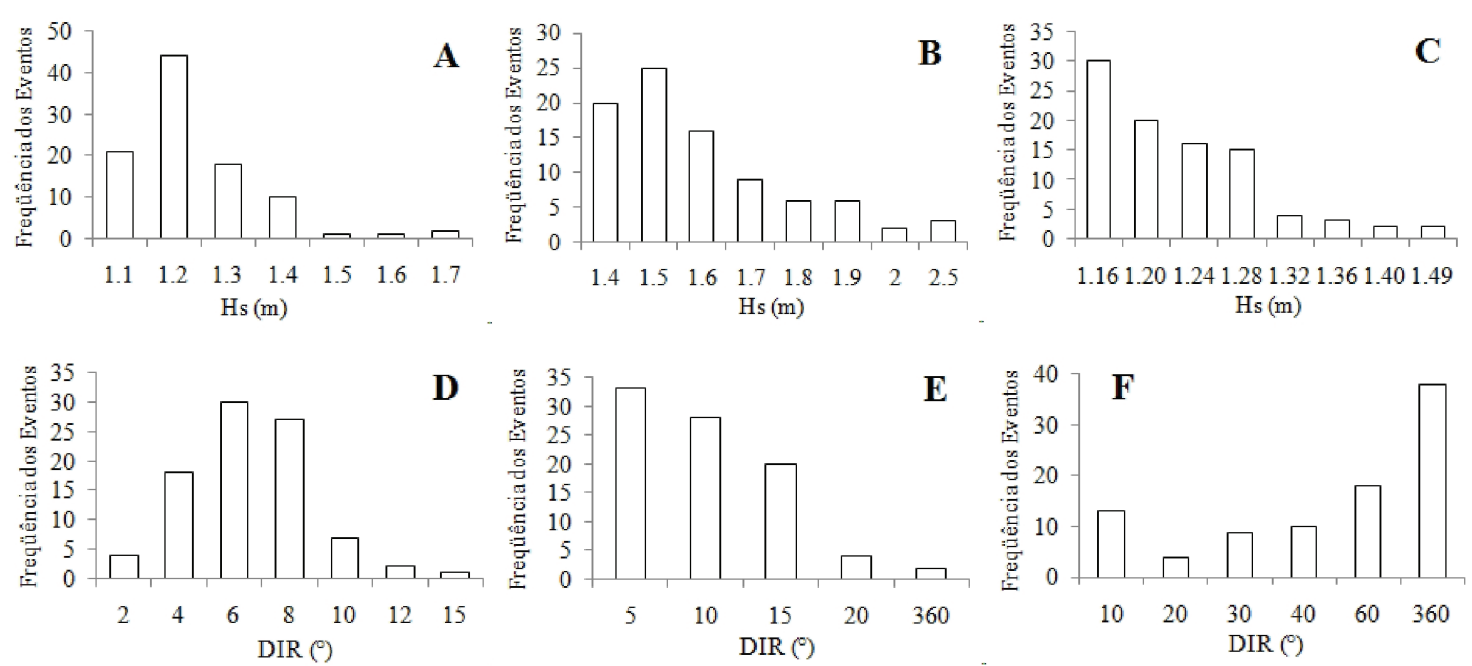

Figura 12. Distribuição das frequências dos eventos de Hs $(\mathrm{m})$ e DIR $\left(^{\circ}\right)$ correspondente ao percentil 99 ao longo da série temporal 2010 - 2015. A) Frequência dos eventos das alturas significativas no PT_1; B) Frequência dos eventos das alturas significativas no PT_2; C) Frequência dos eventos das alturas significativas no PT_3; D) Frequência das direções no PT_1; E) Frequência das direções no PT_2; F) Frequência das direções no PT_3.

Figure 12. Distribution of the frequencies of $\mathrm{Hs}(\mathrm{m})$ and DIR $\left(^{\circ}\right)$ events corresponding of 99 percentile, along the time series 2010 - 2015. A) Frequency of events of significant heights in PT_1; B) Frequency of events of significant heights in PT_2; C) Frequency of events of significant heights in PT_3; D) Frequency of directions on PT_1; E) Frequency of directions on PT_2; C) Frequency of directions on PT_3. 


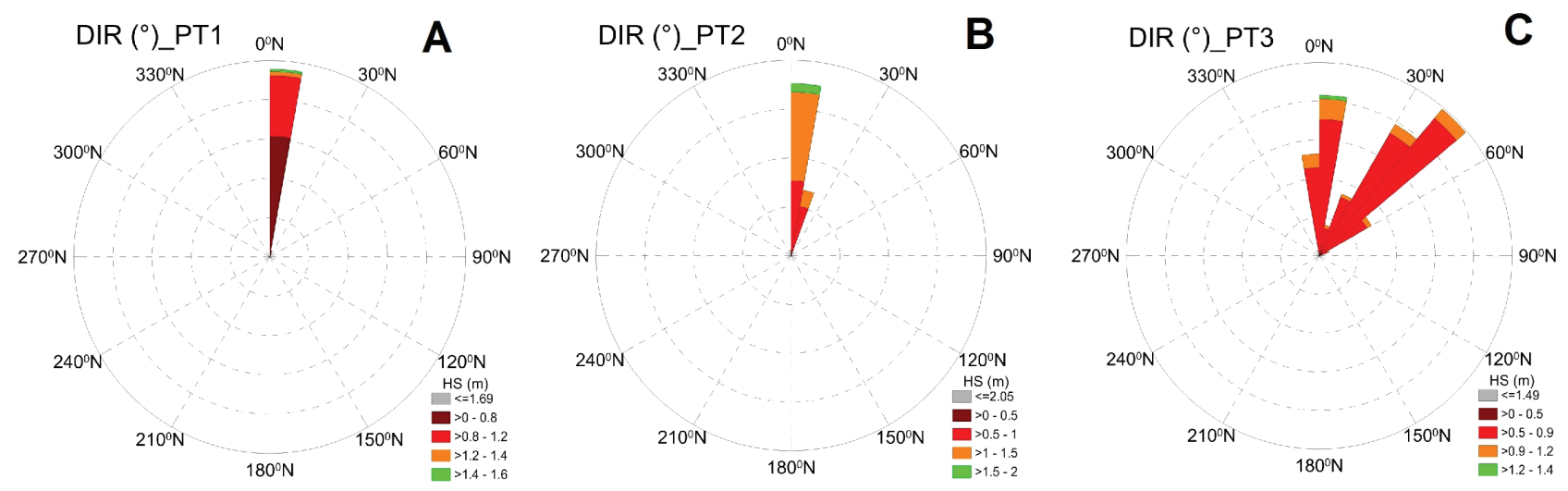

Figura 13. Padrões direcionais dos eventos extremos de ondas para cada estação costeira. A) Padrões direcionais no PT_1; B) Padrões direcionais no PT_2; C) Padrões direcionais no PT_3.

Figure 13.Directional patterns of extreme wave events for each coast station. A) Directional patterns in PT_1; B) Directional patterns in PT_2; C) Directional patterns in PT_3.

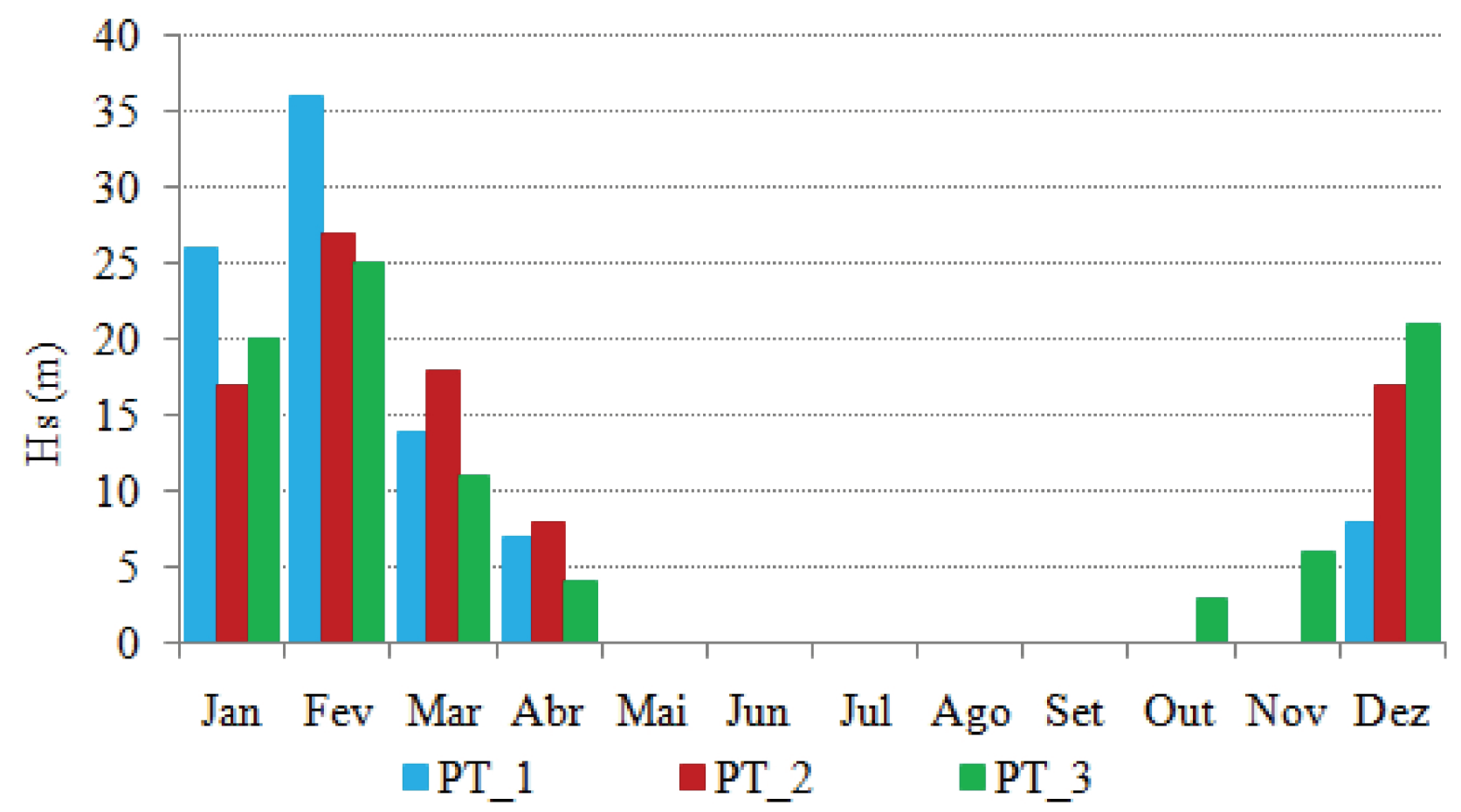

Figura 14. Distribuição de ocorrência dos eventos ao longo dos meses nos PT_1, PT_2 e PT_3.

Figure 14. Occurrence of distribution of events along the months in PT_1, PT_2 e PT_3.

\section{Conclusões}

Neste trabalho, cinco anos (de 2010 a 2015), de características das ondas simuladas foram utilizadas para analisar: a distribuição do domínio espacial anual, sazonal e mensal; a distribuição temporal mensal, sazonal; as variações interanuais e os extremos de ondas na região da plataforma continental do Rio Grande do Norte. As análises estatísticas da série temporal mostraram que os valores máximos das ondas que chegam à costa são: 1,69 m, 2,05 m e 1,48 $\mathrm{m}$. Contudo, as ondas mais frequentes ao longo da série são as que ocorrem entre 0,40 e 0,80 m, com períodos médios de $3 \mathrm{~s}, 5 \mathrm{~s}$ a $7 \mathrm{~s}$, sendo as direções mais frequentes correspondentes as ondas de NE e ENE.

A distribuição anual e sazonal das alturas significativas das ondas no domínio espacial mostra que a zona de maior incidência, com os valores mais elevados está localizada na porção externa da plataforma continental, acima da isóbata de $25 \mathrm{~m}$, com média de 1,96 m e valores máximos acima de $2 \mathrm{~m}$ para Hs com padrão direcional de ENE. 
As distribuições sazonais de Hs e DIR na plataforma continental mostraram que as ondas mais altas são durante o verão (2,5 m) e as ondas de menor valor atingem, sobretudo os meses de inverno $(1,76 \mathrm{~m})$. Em todas as estações a plataforma externa é sempre a zona em que as ondas são mais altas, e muito baixas quando chegam à costa.

Os padrões de direções de acordo com as estações possuem variações distintas, sendo que no verão as características das ondas são predominantes de NNE; no outono os padrões possuem uma característica mais de NE; no inverno, as ondas são fortemente dominadas por padrões de ESE; e na primavera, as ondas são bem distribuídas entre ENE e ESE.

Na distribuição espacial mensal das ondas, a análise confirma que de dezembro a fevereiro as ondas que se propagam na costa são as mais altas ao longo do ano.

Durante a análise da distribuição espacial, foi identificado que no verão e outono, a interação das ondas com o fundo marinho na plataforma continental mostrou-se marcante, pois os processos físicos de refração e difração foram mais evidenciados, sobretudo na transferência do ambiente de águas mais profundas para águas mais rasas. Ao largo as ondas se propagam com direções predominantes de ENE, com maior frequência de ondas de $60^{\circ}$ e $65^{\circ}$; ao entrar na zona intermediária há uma mudança predominante de $\mathrm{NE}\left(45^{\circ}\right)$, ao atingir a costa a maior frequência das ondas varia entre $20^{\circ}$ e $40^{\circ}$, ou seja, de NNE.

As análises das variações temporais para cada estação costeira de medição: PT_1, PT_2 e PT_3, confirmam que durante o verão, as alturas das ondas são as mais altas, com Hs máxima de até $2 \mathrm{~m}$; sendo que nos meses de dezembro a março, as ondas atingem os valores máximos com 2,05 m e valores médios de 1,8 $\mathrm{m}$.

Da mesma forma da análise sazonal e mensal, na análise interanual, os resultados mostraram uma grande variação ao longo dos anos, sobretudo, nos valores máximos de Hs e Tmed. Do período analisado, o ano de 2010 e 2013 mostrou nos resultados uma tendência mais elevada nos valores de $\mathrm{Hs}_{\max }$ nas três estações costeiras, e nos demais anos uma redução significativa respectivamente para as três estações. Nos valores de $\mathrm{Hs}_{\text {med' }^{\prime}}$ houve uma grande diferença entre os valores das estações PT_2 e PT_3 em relação ao PT_1, contudo, a tendência mostrou-se sempre à redução.

Nos valores de período da onda, Tmed ${ }_{\text {max' }}$ os resultados mostram uma tendência similar nas estações do PT_1 e PT_3, sendo nos três primeiros anos, valores de $8 \mathrm{~s}$, reduzindo a partir de 2013. No PT_2, os valores tenderam a oscilar de 2010 reduzindo para 2011, retornando a aumentar para 2013, e a partir deste, tornou a reduzir. Nos valores médios, Tmed, os valores mantiveram-se na média, entretanto, com tendência à diminuição nas três estações.

Nos resultados dos eventos de ondas classificadas acima do percentil 99\%, obtevese um total de 272 eventos distribuídos em: 91 eventos no PT_1; 88 eventos no PT_2; e, 93 eventos no PT_3, sendo que estes eventos significaram apenas 3\% em relação à série temporal. Os eventos mais frequentes ocorrem entre as $\mathrm{Hs}$ de 1,2, 1,5 e 1,1 m, respectivamente, PT_1, PT_2 e PT_3. Esses eventos severos extremos foram mais frequentes em 2010 e 2013 nas três estações, sobretudo em 2013, e ocorrem principalmente, durante o verão e parte do outono, sendo fevereiro o mês das maiores concentrações, 39, 31 e 28\% registradas em cada estação.

Este estudo mostrou que padrões nas características das ondas ao longo de um período de uma série temporal de curto tempo, são importantes para determinar qualquer intervenção em mar ou na zona costeira. Conhecer o comportamento de $\mathrm{Hs}$ anual e interanual, a sazonalidade e os meses na escala espaço-temporal, significa conhecer a época em que os eventos podem ocorrer. Assim, ter o conhecimento das características do clima das ondas, permitirá avaliar a propagação de ondas como recurso energético disponível futuramente.

Agradecimentos. A CAPES, agradecemos o suporto pela bolsa de pós-doutorado à primeira autora do artigo, que permitiu a realização e desenvolvimento do presente trabalho. 


\section{Referências bibliográficas}

Almeida, L.R., Amaro, V.E., Marcelino, A.M.T. \& Scudelari, A.C. 2014. Avaliação do clima de ondas da praia de Ponta Negra (RN, Brasil) através do uso do SMC-Brasil e sua contribuição à gestão costeira. Revista de Gestão Costeira Integrada, 15(2): 135-151.

Battjes, J.A. \& Janssen, J.P.F.M. 1978. Energy loss and set-up due to breaking of random waves. In: PROCEEDINGS OF INTERNATIONAL CONFERENCE ON COASTAL ENGINEERING, 16., 1978, Hamburg. Book of Abstract... Hamburg, ASCE, 569-587.

Booij, N., Ris, R.C. \& Holthuijsen, L.H. 1999. A thirdgeneration wave model for coastal regions, part I, model description and validation. Journal Geophysical Research, 104(4): 76497666.

Busman, D.V., Amaro, V.E. \& Prudêncio, M.C. 2014. Comparison of prognostic models in different scenarios of shoreline position on Ponta Negra beach in Northeastern Brazil. World Academy of Science, Engineering and Technology, 8: 165-169.

Busman, D.V., Amaro, V.E. \& Souza-Filho, P.W.M. 2016. Análise multivariada de métodos de vulnerabilidade física em zonas costeiras tropicais. Revista Brasileira de Geomorfologia, 17(3): 500-516.

Caires, S. 2011. Extreme value analysis: wave data. Geneva, WMO-JCOMM, 38p. (Internal Report, 57). Disponível em: < http://library. wmo.int/pmb_ged/jcomm-tr_57_en.pdf>. Acesso em: 05 abr. 2017.

Campos, R.M. 2009. Análise dos extremos de onda no Rio de Janeiro associados a ciclones extratropicais no Atlântico Sul. Rio de Janeiro, 272p. Dissertação de Mestrado, Programa de Pós-graduação em Engenharia Oceânica, Instituto Alberto Luiz Coimbra, Universidade Federal do Rio de Janeiro.

Campos, R.M. 2014. Análise espacial de eventos extremos de onda usando modelagem numérica. Rio de Janeiro, 314p. Tese de Doutorado. Programa de Pós-graduação em Engenharia Oceânica, Instituto Alberto Luiz Coimbra, Universidade Federal do Rio de Janeiro.
Campos, R.M., Camargo, R. \& Harari, J. 2010. Caracterização de eventos extremos do nível do mar em Santos e sua correspondência com as reanálises do modelo do NCEP no Sudoeste do Atlântico Sul. Revista Brasileira de Meteorologia, 25(2): 175-184.

Candella, R.N. \& Souza, S.M.L. 2013. Ondas oceânicas extremas na costa Sul-Sudeste Brasileira geradas por ciclone com trajetória anormal em maio de 2011. Revista Brasileira de Meteorologia, 28(4): 441-456.

Cardoso Júnior, H.G. 2004. Análise bivariada de extremos de onda e vento na Bacia de Campos. Rio de Janeiro, 125p. Dissertação de Mestrado, Programa de Pós-graduação em Engenharia Oceânica, Instituto Alberto Luiz Coimbra, Universidade Federal do Rio de Janeiro.

Carvalho, J.T. 2010. Simulação da distribuição de energia das ondas oceânicas ao largo do Litoral Brasileiro. São José dos Campos, 169p. Dissertação de Mestrado, Curso de Pós-Graduação em Meteorologia, Instituto Nacional de Pesquisas Espaciais.

Cavalcanti, I.F.A., Ferreira, N.J., Silva, M.G.A.J. \& Dias, M.A.F.S. 2009. Tempo e clima no Brasil. São Paulo, Editora Oficina de textos, 463p.

Chowdhury, S.N. \& Dhawan, S. 2016. Statistical analysis of wave height: the wave energy potential aspect. $7^{\text {st }}$ IEEE International Conference on Power Eletronics, Inteligent Control and System (ICPEICES-2016), 4p.

Chu, P.C., Galanis, G., \& Kuo, Yu-Heng. 2010. Statistical structure of global significant wave heigths. In: CONFERENCE ON PROBABILITY AND STATISTICAS IN ATMOSPHERIC SCIENCES, 20., 2010, Atlanta. Proceedings... Atlanta, p. 1-6.

Coles, S. 2001. An introduction to statistical modelling of extreme values. Springer-Verlag, London, 204p.

Costa, M.B.S.F., Araújo, M., Araújo, T.C.M. \& Sigle, E. 2014. Influence of reef geometry on wave attenuation on a Brazilian coral reef. Geomorphology, 253: 318-327.

CPRM. Companhia de Pesquisa de Recurso Minerais. 2017. Batimetria SRTM 30 Plus V.8.0. Disponível em: <http://www.cprm.gov. br/publique/Geologia/Geologia-Marinha/ 
Projeto-Batimetria-3224.html/>. Acesso em: 08 ago. 2016.

DHN. Diretoria de Hidrografia e Navegação. 2015.

Tábuas de marés. Disponível em: <http:www. dhn.mar.mil.br/>. Acesso em: 15 mar. 2015.

DHN. Diretoria de Hidrografia e Navegação. 2018.

Tábuas de marés. Disponível em: <http:www. dhn.mar.mil.br/>. Disponível em: <https:// www. marinha.mil.br/chm/sites/www. marinha.mil.br.chm/files/dados_de_mare/ macau_2018.pdf>. Acesso em: 11 dez. 2018.

Dreier, N., Schlamkov, C., Fröhle, P., Salecker, D. \& Xu, Z. 2015. Assessment of changes of extreme wave conditions at the German Baltic Sea coast on the basis of future climate change scenarios. Journal of Marine Science and Technology, 23(6): 839-845.

Eldeberky, Y. 1996. Nonlinear transformation of wave spectra in the nearshore zone. Netherlands, 223p. Doctoral Thesis, Civil Engineering and Geoscience, Delft University of Technology.

Farias, E.G.G. \& Souza, J.M.A.C. 2012. Chegada dispersiva de campos de ondas swell na costa oeste do estado do Ceará-Brasil. Arquivos de Ciências do Mar, 45(1): 69-74.

Ferreira, J.A. \& Guedes Soares, C. 1998. An application of peaks over threshold method to predict extremes of significant wave height. Journal of Offshore Mechanics and Arctic Engineering, 120: 165-176.

Fortes C.J.E.M., Pinheiro, L., Santos, J.A. 2011. SOPRO 3.0 - evolução do pacote integrado do SOPRO. Vetor, 21(1): 72-100.

Fortes C.J.E.M., Matos, M.F.A., Scudelari, A.C. \& Amaro, V.E. 2011. Caracterização da agitação marítima nas proximidades da Ponta do Tubarão - RN, Nordeste do Brasil. In: CONGRESSO SOBRE PLANEAMENTO E GESTÃO DAS ZONAS COSTEIRAS DOS PAÍSES DE EXPRESSÃO PORTUGUESA, 6, 2011, Boa Vista. Anais... Cabo Verde: APRH, p. 73.

Guimarães, P.V., Farina, L. \& Toldo Jr., E.E. 2014. Analysis of extreme wave events on the Southern coast of Brazil. Natural Hazards and Earth System Sciences, 14: 3195 - 3205.

Goda, Y., Kudaka, M. \& Kawai, H. 2010. Incorporation of Weibull distribution in $L$-Moments method for regional frequency analysis of peaks-threshold wave heights. In: COASTAL ENGINEERING, 32., 2010, Shanghai, Proceedings... Shanghai, p. 1-11.

Góis, D.M.M., Scudelari, A.C., Rosman, P.C.C. \& Amaro, V.E. 2009. Um estudo sobre propagação de manchas de óleo na zona costeira de Galinhos e Guamaré - RN. In: SIMPÓSIO BRASILEIRO DE RECURSOS HÍDRICOS, 18., 2009, Campo Grande. Anais... Campo Grande: $\mathrm{ABRH}$.

Gomes, L.R.S. 2017. Simulação numérica da incidência de ondas marítimas em praias de Natal/RN, Nordeste do Brasil, 2012 a 2014. Natal, 93p. Dissertação de Mestrado. Programa de Pós-graduação em Ciências Climáticas, Universidade Federal do Rio Grande do Norte.

Hasselmann, K., Barnett, T.P., Bouws, E., Carlson, H., Cartwright, D.E., Enke, K., Ewing, J.A., Gienapp, H., Hasselmann, D.E., Kruseman, P., Meerburg, A., Miller, P., Olbers, D.J., Richter, K., Sell, W. \& Walden, H. 1973. Measurements of wind wave-growth and swell decay during the Joint North Sea Wave Project (JONSWAP), Ergnzungsheft Deutschen Hydrographischen Zeitschrift Reihe, A8(12): 1-95.

Hasselmann, S., Hasselmann, K., Allender, J.H. \& Barnett, T.P. 1985. Computations and parameterizations of the linear energy transfer in a gravity wave spectrum, Part II: parameterizations of nonlinear transfer for application in wave models. Journal of Physical Oceanography, 15(11): 1378-1391.

Holthuijsen, L.H. 2007. Waves in oceanic and coastal waters. Cambridge, Cambridge University Press, 387p.

INMET. Instituto Nacional de Meteorologia. 2013. Dados das séries temporais climáticas da Rede do INMET. Disponível em: <http://www. inmet.gov.br/>. Acesso em: Abr 2013.

Izaguirre, C., Méndez, F.J., Menéndez, M. \& Losada, I.J. 2011. Global extreme wave height variability based on satellite data. Geophysical Research Letters, 38: 1-6.

Kahma, K., Hauser, D., Krogstad, H.E., Lehner, S., Monbaliu, J. Wyatt, L.R. 2005. Measuring and analysing directional spectra of ocean waves. COST Action 174, EUR 21367, Brussels, 15p. Kirby, J.T. \& Dalrymple, R.A. 1994. REF/DIF 1 
version 2.5 Documentation and user's manual Newark, CACR, 122p. (Internal Report, 94-22).

Komen, G.J., Hasselmann, S. \& Hasselmann, K. 1984. On the existence of a fully developed wind sea spectrum. Journal of Physical Oceanography, 14: 1271-1285.

Malmann, D.L.B., Pereira, P.S., Nogueira, P.F.R.S.M. \& Santos, F.M.M. 2014a. Classificação morfodinâmica das praias arenosas de Ipojuca (Pernambuco, Brasil) através da análise semântica de imagens de satélite pancromática. Pesquisas em Geociências, 41: 169-189.

Malmann, D.L.B., Araújo, T.C.M. \& Droguett, E.L. 2014b. Caracterização do litoral de Pernambuco (Brasil) quanto ao processo erosivo em curto e médio-prazo. Quaternary and Environmental Geosciences, 5: 137-154.

Mathiesen, M., Goda, Y., Hawkes, P.J., Mansard, E., Martin, M.J., Peltier, E., Thompson, E.F. \& Van Vledder, G. 1994. Recommended practice for extreme wave analysis. Journal of Hidraulic Research, 32(6): 803-814.

Martucci, G., Carniel, S., Chiggiato, J., Sclavo, M., Lionello, P. \& Galati, M.B. 2010. Statistical trend analysis and extreme distribution of significant wave height from 1958 to 1999 - an application to the Italian Seas. Ocean Science, 6: 525-538.

Matos, M.F.A. \& Amaro, V.E. 2011. Oscillatory coastal wave and currents profile in the Northern Continental Shelf of Rio Grande do Norte State, Northeast Brazil. In: INTERNATIONAL GEOSCIENCE PROGRAMME, 5, 2011, Sidney. Abstract... Sidney: UNESCO/ IUGS/Project 526, 8p.

Matos, M.F.A., Fortes, C.J.E.M., Amaro, V.E. \& Scudelari, A.C. 2013. Comparative analysis of agitation obtained the numeric model (SWAN) in modelling Rio Grande do Norte (Brazil) Northern Coastal waves and field data. Journal of Integrated Coastal Zone Management, 13(3): 283-299.

Matos, M.F.A., Scudelari, A.C., Amaro, V.E. \& Fortes, C.J.E.M. 2017. Integração de modelagem numérica (SWAN) e dados de campo na determinação do clima de ondas no litoral setentrional do Rio Grande do Norte. Revista Brasileira de Geomorfologia, 18(2): 311-328.
Parente, C.E., Lima, J.A., Violante-Carvalho, N. \& Assunção, C.B. 2001. Wave and wind extremes values in good weather situations in the Campos Basin of Rio de Janeiro. In: PROCEEDINGS OF 20TH INTERNATIONAL CONFERENCE ON OFFSHORE MECHANICS AND ARTIC ENGINEERING, 20, 2001, Rio de Janeiro. Book of Abstract... Rio de Janeiro, OMAE (CD-ROM).

Pedersen, T., Siegel, E. \& Wood, J. 2007. Directional wave measurements from a subsurface bouy with an acustic wave and current profile (AWAC). In: OCEANS EUROPE, 3., 2007, Aberdeen, Proceedings... Aberdeen, p. 1-10.

Pinho, U.F. 2003. Caracterização dos estados de mar na Bacia de Campos. Rio de Janeiro, 137p. Dissertação de Mestrado, Programa de Pós-graduação em Engenharia Oceânica, Instituto Alberto Luiz Coimbra, Universidade Federal do Rio de Janeiro.

Resio, D.T. \& Vincent, C.L. 1977. Estimation of winds over the Great Lakes. J. Waterway, Prot Coastal and Ocean Eng. ASCE, 103: 265-285.

Ris, R.C. 1997. Spectral modelling of wind waves in coastal areas. Netherlands, 180p. Doctoral Thesis, Civil Engineering and Geoscience, Delft University of Technology.

Roscoe, K., Caires, S., Diermanse, F. \& Groeneweg, J. 2010. Extreme offshore wave statistics in the North Sea. WIT Transactions on Ecology and the Environment, 133: 47-58.

Rosman, P.C.C. 2006. Referências Técnicas do SisBAHIA - Sistema de Base de Hidrodinâmica Ambiental. Programa de Engenharia Oceânica, Área de Engenharia Costeira e Oceanográfica, COPPE/UFRJ, Rio de Janeiro, 367p. (Referência Técnica).

Rosso, T.C.A. 2007. Gestão integrada em bacias hidrográficas costeiras. In:WORKSHOP SOBRE HIDRODINÂMICA COSTEIRA - AMIGOS DE BOUSSINESQ, 1, 2007, Vitória. Anais... Vitória: UFRJ, v. 1, p. 1-7.

Salcedo-Castro, J., Silva, N.P., Camargo, R., Marone, E. \& Sepúlveda, H.H. 2018. Estimation of extreme wave heights return period from short-term interpolation of multi-mission satellite data: application to the South Atlantic. Ocean Science, 14(4): 911-921.

Santos, A.J., Fortes, C.J.E.M., Pinheiro, L. \& Neves, 
M.G. 2005. A software package for wave characteristics in ports. Laboratório Nacional de Engenharia Civil - LNEC. Lisboa, 20p.

Scudelari, A.C., Fortes, C.J.E.M. \& Dantas, A.L.S.S. 2007. Propagação da agitação marítima na região de Galinhos-Guamaré, Rio Grande do Norte, Brasil. In: CONGRESSO IBERO LATINO-AMERICANO SOBRE MÉTODOS COMPUTACIONAIS EM ENGENHARIA, 7, 2007, 1, Porto. Anais... Porto: CMNE/CILANCE, p. 1-18.

Scudelari, A.C., Neves, C.F., Amaro, V.E. \& Santos Júnior, O.F. 2016. Ponta Negra Beach, Natal, Brazil: A Roll and Rock Story. Journal of Coastal Research, 75, p. 815-819.

Semedo, A., Suselj, K., Rutgersson, A. \& Sterl, A. 2011. A global view on the wind sea and swell climate and variability from ERA-40. Journal of Climate, 24: 1461-1479.

Silva, P.E.D. 2013. Caracterização do padrão de ondas na costa do Brasil por meio de modelagem numérica. São José dos Campos, 231p. Dissertação de Mestrado, Instituto Nacional de Pesquisas Espaciais.

Singh, P. 2006. A statistical analysis of extreme wave heights in the North Sea. Netherlands, 133p. Doctoral Thesis, Civil Engineering and Geoscience, Delft University of Technology.

Soares, C.G., Bitner-Gregersen, E.M., Antão, P. 2001. Analysis of the frequency of ship accidents under severe North Atlantic weather conditions. Design and Operation for Abnormal Conditions, The Royal Institution of Naval Architects, p. 221-230.

Spindola, R.L. \& Araújo, A.M. 2017. Wave energy resource of Brazil: an analysis from 35 years of ERA-Interim reanalysis data. PLoS One, 12: 0183501.

Spindola, R.L., Andrade, P.V.F. \& Araújo, A.M. 2016. Theoretical analysis mechanical energy conversion by a point absorver WEC using reanalysis wave data. In: INTERNATIONAL CONFERENCE ON MARITIME TECHNOLOGY AND ENGINEERING-MARTECH 2016, 3, 2016, Lisbon. Book of Abstract... Lisbon, p. 1103-
1109.

Teena, N.V., Kumar, S., Sudheesh, K. \& Sajeev. R. 2012. Statistical analysis on extreme wave height. Natural Hazards, 64(1): 223-236.

USGS. United States Geological Survey. 2016a.

Data set. SRTM 1 Arc-Second Global. Disponível em: <http://earthexplorer.usgs. gov>. Acesso em: 15 ago. 2016.

USGS. United States Geological Survey. 2016b. Data set. Landsat 8 OLI/TIRS C1 Level-1. Disponível em: <http://earthexplorer.usgs. gov>. Acesso em: 15 abr. 2016.

Van Vledder, G. 2006. The WRT method for the computation of non-linear four wave interactions in discrete spectral wave models. Coastal Engineering, 53: 223-242.

Van Gelder, P., De Ronde, J., Neykov, N.M. \& Neytchev, P. 2000. Regional frequency analysis of extreme wave heights: trading space for time. In: COASTAL ENGINEERING, 27., 2000, Sydney. Proceedings... Sydney. p. 1099-1112. Disponível em: <https://ascelibrary.org/doi/ pdf/10.1061/40549(276)85.pdf >. Acesso em: 30 mai. 2017.

Vital, H., Stattegger, K., Amaro, V.E., Schwarzer, K., Frazão, E.P. \& Tabosa, W.F. 2008. A modern high-energy siliciclastic-carbonate platform: Brazilian Continental Shelf Adjacent to Northern Rio Grande do Norte State, NE Brazil. In: Hampson, G.J., Steel, R.J., Burgess, P.M., Dalrymple, R.W. (Ed.). Recent Advanced in Models of Siliciclastic Shallow-Marine Stratigraphy. Tulsa, Society for Sedimentary Geology, p. 175-188.

Vicente Júnior, C. \& Pereira, P.S. 2017. Aspectos sobre o estado da arte de estudo da zona de espraiamento. Revista Brasileira de Geomorfologia, 18(2): 197-223.

Wilks, D.S. 2006. Statistical Methods in the Atmospheric Sciences. Amsterdam, Elsevier Academic Press, 627p.

Young, I.R. 1999. Seasonal variability of the global ocean wind and wave climate. International Journal of Climatology, 19: 931-950. 